\title{
The Iwex Spectrum
}

\author{
P. Müller and D. J. Olbers \\ Institut für Geophysik, Universität Hamburg, Hamburg, West Germany \\ Max-Planck-Institut für Meteorologie, Hamburg, West Germany
}

\section{J. WILLEBRAND ${ }^{1}$}

Institut für Meereskunde an der Universität Kiel, Kiel, West Germany

\begin{abstract}
We describe the analysis of current and temperature cross spectra from the trimoored internal wave experiment Iwex and present a spectral model for the fluctuations in the internal wave range. The model consists of internal waves contaminated by temperature and current fine structure and by current noise. Except for the inertial and tidal frequencies the distribution of wave energy is vertically symmetric and horizontally isotropic. The wave number spectrum is characterized by a bandwidth of about $20-10$ equivalent modes (decreasing with frequency), by a power law at high wave numbers, and by a sharp peak at low wave numbers. The general features of this spectrum agree fairly well with the one proposed by Garrett and Munk (1975). The contaminations increase with frequency. Contamination by temperature fine structure is confirmed by independent measurements. The Iwex spectral model provides a consistent description of all observed cross spectra.
\end{abstract}

\section{INTRODUCTION}

This paper describes the analysis of current and temperature data from the trimoored internal wave experiment Iwex and presents a spectral model of the observed fluctuations in the internal wave range. These fluctuations are generally interpreted as a random superposition of free linear internal waves. Their energy spectrum in wave number-frequency space has never been measured. The various measurements by moored [e.g., Fofonoff, 1969; Webster, 1972], towed [e.g., Katz, 1975], and dropped [e.g., Millard, 1972; Cairns, 1975; Sanford. 1975] instruments only provide incomplete information. The first attempt to estimate the complete energy spectrum was made by Garrett and Munk [1972]. Combining measurements from different locations, depths, times, and instruments, they constructed a model spectrum which is believed to reflect the principal features of the internal wave field in the ocean. Their model, which comprises a considerable amount of internal wave observations, determines the characteristic space-time scales, suggests isotropy and symmetry, and points toward some universality of the spectrum. While the use of an inhomogeneous data set allows the determination of the mean internal wave field and its geophysical variability, it does not allow the determination of the local structure. It has already been noted by Garrett and Munk [1972] that measurements of the internal wave field may be contaminated by current and temperature fine structure, by Doppler shift, by small-scale turbulence, and by mooring motion. In particular, the contamination by temperature fine structure has been studied extensively [Phillips, 1971; Garrett and Munk, 1971; McKean, 1974; Joyce, 1974].

The work of Garrett and Munk initiated various efforts to refine their model and overcome its limitations and shortcomings (cf. internal wave papers in Journal of Geophysical Research, vol. 80, 1975). One of the major efforts was Iwex. This experiment consisted of a three-dimensional array of 20 current meters and temperature sensors deployed in the main thermocline. The high spatial resolution was conceived as necessary to determine quantitatively the wave num-

\footnotetext{
${ }^{1}$ Now at Geophysical Fluid Dynamics Program, Princeton University, Princeton, New Jersey 08540.

Copyright (C) 1978 by the American Geophysical Union.
}

ber-frequency spectrum of the internal wave field. The simultaneous measurement of horizontal current and temperature is necessary to detect possible contaminations. The experiment was supplemented by CTD profiles at the beginning and end of the experiment [Hayes et al., 1975]. The Iwex data set and preliminary results have been discussed by Briscoe [1975]. Various specific aspects have been investigated. Stationarity of the field was analyzed by C. Frankignoul and T. M. Joyce (1977), Gaussianity by Briscoe [1977], temperature fine structure by Joyce and Desaubies [1977], turning point effeets by Desaubies [1975], and the $M_{2}$ tide by Noble and Joyce [1977].

In this paper we use the current and temperature cross spectra to determine the basic constituents of the observed motion and to model their wave number-frequency structure. In the first part, basic results are inferred from selected data by simple reasoning. This analysis reveals the main constituents of the field: internal waves contáminated by temperature and current fine structure and current noise. It also provides order of magnitude estimates for various parameters describing the spatial structure of the fields.

In the second part we construct a spectral model for the complete data set. This requires the generalization and formalization of the previous analysis so that all data can be considered simultaneously. The analysis proceeds in two steps: consistency tests and inverse analysis. Consistency tests [Müller and Siedler, 1976] establish basic properties of the motion, e.g., deviations from isotropy or from a pure internal wave field. They generalize concepts introduced by Fofonoff [1969]. The inverse analysis [Wiggins, 1972; Schott and Willebrand, 1973] determines parameters of the spectrum by least squares fit. The resulting parameters are displayed in Figure 15. This spectral model provides a consistent description of the complete Iwex data set.

The algebraic structure of internal waves which is needed for all parts of the analysis is reviewed in the appendix. This paper is a summary of the report by Willebrand et al. [1977] (henceforth referred to as paper 1).

\section{DAta SET}

The Iwex field experiment was performed for 42 days in 1973 at a site $\left(27^{\circ} 44^{\prime} \mathrm{N}, 69^{\circ} 51^{\prime} \mathrm{W}\right)$ in the Sargasso Sea. The instrument array was supported by an extremely stable three- 
legged mooring with the form of a tetrahedron. Current meters and temperature sensors were attached to the three legs on eight horizontal levels between $604-\mathrm{m}$ and $2050-\mathrm{m}$ depths. The vertical distance between consecutive levels increased from top to bottom in a logarithmic manner. Figure 1 illustrates the geometry of the array and displays the profile of the buoyancy frequency $N(z)$ (in the deepwater column below $2500 \mathrm{~m}, N$ is almost constant, $N_{d w}=0.36 \mathrm{cph}$ ). The array was mainly instrumented with vector averaging current meters (VACM), which were modified to include differential temperature measurements; i.e., temperature $T$ and temperature difference $\Delta T$ over a vertical distance of $1.74 \mathrm{~m}$ were measured. The temperature time. series were used to estimate the vertical displacement by

$$
\zeta(t)=-T(t)\left(\frac{\overline{\Delta T}}{1.74 \mathrm{~m}}\right)^{-1}
$$

The bar denotes the time average over the entire period of the experiment. A detailed description of the experiment, the data, and the estimation of cross spectra has been given by Briscoe [1975] and Tarbell et al. [1976].

Equivalent to $\zeta$ we will frequently use the vertical velocity

$$
u_{0}=\dot{\zeta}
$$

Instead of the horizontal Cartesian components $u_{1}$ and $u_{2}$ of the current we will use the rotary components

$$
u_{ \pm}=\frac{1}{(2)^{1 / 2}}\left(u_{1} \pm i u_{2}\right)
$$

which lead to a more convenient representation of the kinematical structure of internal waves.

The cross spectrum between the component $u_{u}$ at the $i$ th position and the component $u_{v}$ at the $j$ th position is denoted by

$$
A_{\mu \nu}{ }^{i j}(\omega)=P_{\mu \nu}{ }^{i j}(\omega)-i Q_{\mu \nu}{ }^{i j}(\omega)
$$

where $P_{\mu \nu}{ }^{2 j}$ is the cospectrum and $Q_{\mu \nu}{ }^{\nu}$ is the quadspectrum. Covariances of cross spectra are estimated following, e.g., Jenkins and Watts [1968]:

$$
\begin{aligned}
\operatorname{Cov}\left[P_{\alpha \beta}{ }^{l \jmath}, P_{\mu \nu}{ }^{k \prime}\right]=\frac{1}{\nu}\left(P_{\alpha \nu}{ }^{l i} P_{\beta \mu}{ }^{j k}+\right. & Q_{\alpha \nu}{ }^{l l}{Q_{\beta \mu}}{ }^{k} \\
& \left.+P_{\alpha \mu}{ }^{l k} P_{\beta \nu}{ }^{\prime \prime}+Q_{\alpha \mu}{ }^{k k} Q_{\beta \nu}{ }^{\prime \prime}\right)
\end{aligned}
$$

$\operatorname{Cov}\left[P_{\alpha \beta}{ }^{l j}, Q_{\mu \nu}{ }^{k \prime}\right]=\frac{1}{\nu}\left(P_{\beta \mu}{ }^{k} Q_{\alpha \nu}{ }^{l l}-P_{\alpha \nu}{ }^{l}{ }^{\prime} Q_{\beta \mu}{ }^{k k}\right.$

$$
\left.-P_{\beta \nu}{ }^{l} Q_{\alpha \mu}{ }^{l k}+P_{\alpha \mu}{ }^{i k} Q_{\beta \nu}{ }^{l}\right)
$$

$\operatorname{Cov}\left[Q_{\alpha \beta^{i j}}, Q_{\mu \nu}{ }^{k l}\right]=\frac{1}{\nu}\left(P_{\alpha \mu}{ }^{k k} P_{\beta \nu}{ }^{l l}-P_{\alpha \nu}{ }^{\prime l} P_{\beta \mu}{ }^{j k}\right.$

$$
\left.+Q_{\alpha \mu}{ }^{k k} Q_{\beta \nu}{ }^{l l}-Q_{\alpha \nu}{ }^{l l} Q_{\beta \mu}{ }^{j k}\right)
$$

Here $\nu$ is the equivalent number of degrees of freedom for the autospectral estimate. Values for $\nu$ are calculated from Nuttal [1971] and Perrson [1974].

Coherence and phase are defined by

$$
\begin{aligned}
& \gamma_{\mu \nu}{ }^{l j}=\left|A_{\mu \nu}{ }^{l \nu}\right|\left(P_{\mu \nu}{ }^{i} P_{\nu \nu}{ }^{j J}\right)^{-1 / 2} \\
& \Phi_{\mu \nu}{ }^{j}=\operatorname{Arctan}\left(Q_{\mu \nu}{ }^{\prime \prime}, P_{\mu \nu}{ }^{l j}\right)
\end{aligned}
$$

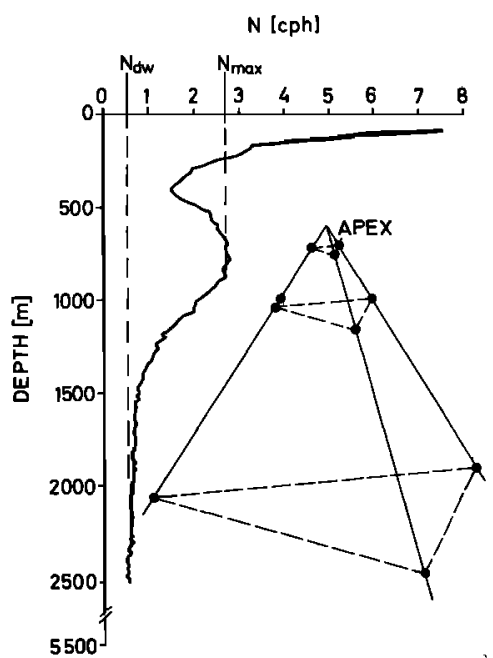

Fig. 1. Profile of buoyancy frequency $N(z)$ at the Iwex site. The geometry of the Iwex array is schematically indicated. Points are instrument positions. Near the apex, 10 more instruments (not shown) are located.

Confidence intervals are calculated following Amos and Koopmans [1963] and Goodman [1957]. All coherence figures show the $95 \%$ confidence level for zero true coherence. Error bars always give the standard deviation.

Our analysis is restricted to frequencies between the inertial frequency $f=0.03878 \mathrm{cph}$ and the maximal buoyancy frequency $N_{\max }=2.76 \mathrm{cph}$ in the main thermocline. In this interval, cross-spectral estimates have been computed at 28 frequency points which are almost equispaced on a logarithmic axis. In the figures the frequency axis is always logarithmically displayed, and the frequency points are numbered from 3 to 30 . The third frequency, $\omega_{s}=0.040 \mathrm{cph}$, is slightly larger than $f$; the sixth frequency, $\omega_{6}=0.080 \mathrm{cph}$, is slightly less than the frequency $M_{2}=0.08052 \mathrm{cph}$ of the semidiurnal tide. For instruments in the deeper levels the high frequencies are beyond the local buoyancy frequency. Corresponding data are omitted from the analysis. This applies to the deepest level (at $2050 \mathrm{~m}$ ) for frequencies larger than $\omega_{22}=0.660 \mathrm{cph}$ and the three deepest levels (below $1000 \mathrm{~m}$ ) for frequency $\omega_{\mathrm{so}}=1.947$ cph.

In this paper we only consider cross spectra which are averaged over the entire period of the experiment. We only included sensors which gave high-quality data for the entire period, leaving us with data from 20 temperature sensors and 9 current meters. This yields 38 autospectra and 1406 cospectra and quadspectra. The equivalent number $\nu$ of degrees of freedom increases from $O(50)$ near $f$ to $O(300)$ near $N_{\max }$ (for details, cf. Figure 13a). The spatial separations between the temperature sensors range from $2 \mathrm{~m}$ to $O(1500 \mathrm{~m})$ horizontally as well as vertically; the current meters cover a smaller interval from $7 \mathrm{~m}$ to $O(500 \mathrm{~m})$.

\section{Preliminary Analysis}

In interpreting deep-sea current or temperature measurements it is generally assumed that the motions in the frequency band between inertial and local buoyancy frequency can be attributed to random linear internal waves. There are two representations of random internal wave fields which primarily differ with respect to their statistical conceptions. In the standing mode representation, vertically upward and downward propagating wave components (with the same frequency and 
horizontal wave vector) have equal amplitude and a fixed phase relation, thus forming a mode. In the propagating wave representation the amplitudes and phases are independent and the vertical structure is approximated by WKB solutions. Which of these descriptions is more appropriate must be inferred from our data set. To be definite, we start with the conceptually simpler and more convenient WKB description.

The cross-spectral matrix of a random internal wave field can be rigorously expressed in terms of the complete energy spectrum which describes the distribution of wave energy in wave number-frequency space. In the propagating wave representation we find (cf. appendix)

$$
\begin{array}{r}
I_{\mu \nu}{ }^{\prime \jmath}(\omega)=\sum_{\sigma= \pm} \int_{0}^{\infty} d \alpha \int_{0}^{2 \pi} d \varphi E^{\sigma}(\alpha, \varphi, \omega) U_{\mu \nu}^{i j}(\varphi, \omega, \sigma) \\
\cdot \exp \left[-i\left(\alpha \cdot \mathbf{r}_{l j}+\sigma \theta_{l \jmath}\right)\right.
\end{array}
$$

(Symbols are explained in the notation section; the kernel $U_{\mu \nu}{ }^{3}$ is the component-by-component product of the matrices (A 21), (A22), and (A23) given in the appendix). The energy spectrum $E^{\sigma}(\alpha, \varphi, \omega)$ consists of the two branches for upward ( $\sigma$ negative) and downward ( $\sigma$ positive) propagating energy. If these are equal, the wave field will be called (vertically) symmetric. If the spectrum is independent of the direction $\varphi$ of the horizontal wave vector $\alpha$, we will speak of (horizontal) isotropy. The eigenvector matrix $U_{\mu v}{ }^{l j}$ is defined such that $E^{\sigma}(\alpha, \varphi, \omega)$ is the distribution of total energy per unit surface area. The vertical distribution of wave energy can be inferred from it by WKB theory.

We shall utilize relation (7) extensively in two ways. The structure of $U_{\mu \nu}{ }^{\prime \prime}$ implies certain relations among the different components of the cross-spectral matrix. These consistency relations will be used to decide whether or not the observed data may be described by internal wave kinematics. Furthermore, the reciprocal relation between the wave number distribution of the energy and the spatial structure of the cross spectra will be used to determine features and characteristic parameters of the energy spectrum.

\section{Energy Level}

The Iwex autospectra show properties already observed in earlier experiments: peaks at the inertial and tidal frequencies followed by an almost -2 slope in the internal wave contin- uum. A small hump near the local buoyancy frequency is found in the displacement spectra. This may be attributed to phase locking near the turning point [Desaubies, 1975].

The autospectra scale according to WKB theory, as demonstrated by Briscoe [1975]. Hence we may consider vertically scaled and averaged spectra. Figure $2 a$ shows the average rotary autospectra $P_{++}$and $P_{--}$of the anticlockwise and clockwise components of the horizontal current, and Figure $2 b$ shows the average vertical displacement spectrum $\boldsymbol{P}_{\zeta \zeta}$. Notice the smallness of the error bars, which demonstrates the high overall accuracy of the experiment. At low frequencies the energy is almost entirely confined to the clockwise spectrum $P_{--}$. In contrast to $P_{--}$, the anticlockwise spectrum $P_{++}$ contains a significant amount of tidal energy at $M_{2}$. This is presumably due to the counterclock wise rotation of the barotropic cotidal lines in the northwest Atlantic [Zetler et al., 1975]. The spectrum of vertical displacement shows a marked $M_{2}$ tidal peak associated with baroclinic wave energy. All three spectra, in particular those of the horizontal current, have a smooth behavior in the wave continuum. Even near the deepwater buoyancy frequency $N_{d w}$ we find no changes in the general decrease. We may conclude that there must be strong local saturation processes governing the dynamical balance of the spectrum.

If the observed fluctuations are due to a random ensemble of linear internal waves, the three spectra $\boldsymbol{P}_{++}, \boldsymbol{P}_{--}$, and $\boldsymbol{P}_{\zeta \zeta}$ are related by [Fofonoff. 1969] (cf. also the appendix)

$$
\frac{P_{++}(\omega)}{P_{--}(\omega)}=\left(\frac{\omega-f}{\omega+f}\right)^{2}
$$

and

$$
\frac{1}{2} \frac{N^{2} P_{\zeta \zeta}(\omega)}{P_{++}(\omega)+P_{--}(\omega)}=\frac{1}{2} \frac{N^{2}}{\omega^{2}+f^{2}} \frac{\omega^{2}-f^{2}}{N^{2}-\omega^{2}}
$$

These ratios are displayed in Figure 3. In the continuum (i.e., $\omega$ $>M_{2}$ ), relation (8) is fairly well satisfied, while at $f$ it is strongly violated.

The violation of (8) at $\omega=f$ can simply be ascribed to the finite frequency resolution of the experiment. Our estimate $P_{\nu v}(\omega)$ is the average of the true spectrum over a finite frequency interval. If the true clockwise spectrum behaves smoothly at $\omega$, the observed value of the anticlockwise spec-

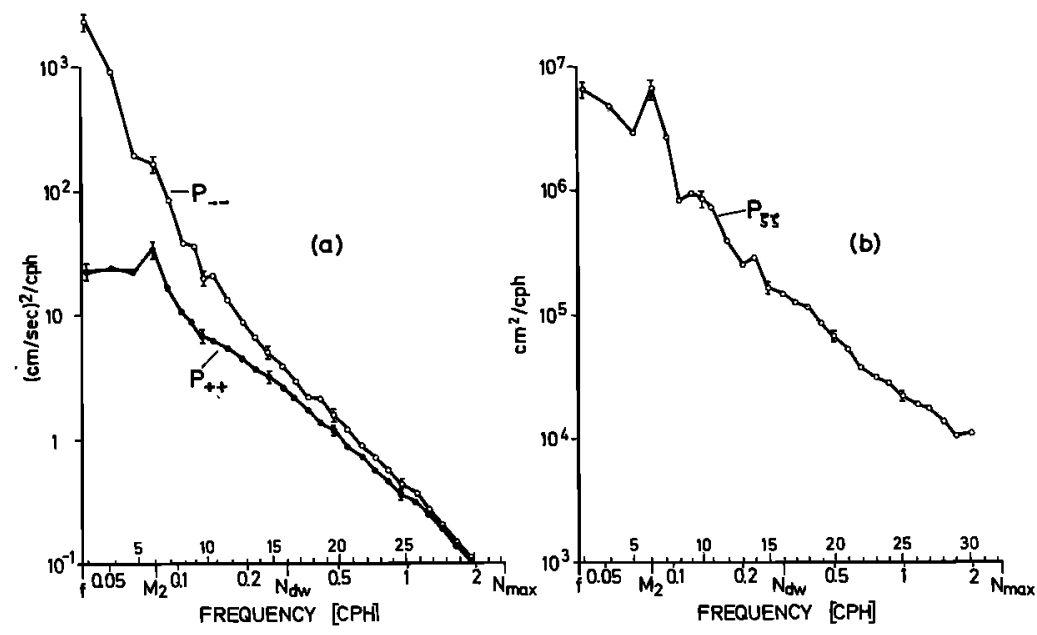

Fig. 2. Average spectra scaled to depth of $604 \mathrm{~m}:(a)$ the rotary spectra $P_{--}$(clockwise) and $P_{++}$(anticlockwise) and $(b)$ the displacement spectrum $P_{\zeta \zeta}$. Error bars are rms deviations. 

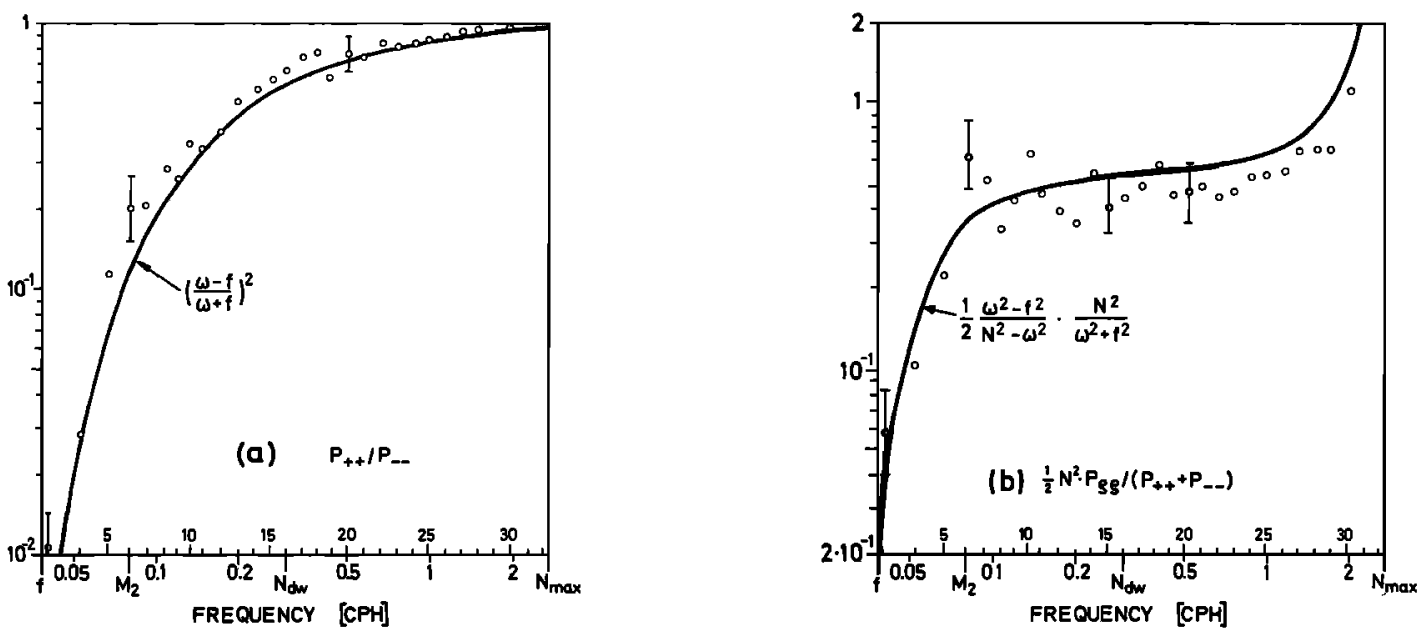

Fig. 3. Internal wave energy tests. (a) Observed ratio $P_{++} / P_{--}$of average rotary spectra and the theoretical curve (8). (b) Observed ratio $P_{\zeta \zeta} /\left(P_{++}+P_{--}\right)$and the theoretical curve (9).

trum is approximately

$$
P_{++}(\omega) \approx P_{--}(\omega) \frac{1}{2 \Delta} \int_{\omega-\Delta}^{\omega+\Delta} d \omega^{\prime}\left(\frac{\omega^{\prime}-f}{\omega^{\prime}+f}\right)^{2}
$$

which yields $P_{++} / P_{--} \approx \Delta^{2} /\left(12 f^{2}\right)$ at $\omega=f$. At low frequencies the resolution is $\Delta \approx f / 3$, so that $P_{++} / P_{--} \approx 10^{-2}$ as observed. The discrepancy at $M_{2}$ cannot be attributed to the finite resolution. Since (8) applies to modes as well, the observed motions cannot be described by free random internal waves. Forced and/or deterministic wave components may be a more suitable description [Hendry, 1975].

The observed ratio $\boldsymbol{P}_{\zeta \zeta} /\left(\boldsymbol{P}_{++}+\boldsymbol{P}_{--}\right)$lies below the theoretical value almost everywhere in the continuum. Here the total energy computed from horizontal current exceeds the one computed from the displacement by about $10 \%$ almost systematically. This feature will be referred to as energy disparity.

\section{Coherence Disparity}

The coherences between the same components of the motion contain almost all information about the spatial scales and the spectral shape of the observed fluctuations. In addition they allow some simple tests of the internal wave assumption which are similar to the energy tests discussed above.

Figure 4 displays the same component coherences for a slant and a horizontal separation. All coherences show an almost monotonical decrease with frequency. The most striking feature is the different behavior of horizontal currents and vertical displacements. According to internal wave theory, the same component coherences should be equal for all separations and frequencies (cf. equation (A30)):

$$
\gamma_{++}=\gamma_{--}=\gamma_{00}
$$

While the horizontal currents satisfy the relation $\gamma_{++}=\gamma_{--}$, both coherences are significantly lower than $\gamma_{00}$. The difference increases with frequency and is larger $(\approx 30 \%)$ for slant than for purely horizontal separations $(\approx 10 \%)$. This coherence disparity is a general feature of the Iwex data set (cf. also Briscoe [1975]). Since (11) holds in the average also for standing modes, the data cannot be explained by pure internal waves.

To study this disparity in more detail, we compare the spatial structure of the observed coherences with the structure expected for a pure internal wave field. For simplicity, consider an isotropic and symmetric energy spectrum $E(\alpha, \omega)$. The coherence (strictly speaking, the normalized cospectrum) between two sensors separated horizontally by $r$ and vertically by $\Delta z$ is given by (cf. equation (A40))

$$
\begin{aligned}
\gamma(r, \Delta z)=\int_{0}^{\infty} d \alpha E(\alpha, \omega) J_{0}(\alpha r) \cos (\beta \Delta z) \\
\cdot\left[\int_{0}^{\infty} d \alpha E(\alpha, \omega)\right]^{-1}
\end{aligned}
$$

For purely horizontal separations the coherence is the Hankel transform of the energy spectrum. Since most slant angles of the Iwex array are rather steep compared to the slope of the wave characteristics, the slant coherence behaves like a purely vertical coherence which is the cosine'transform of the energy spectrum. Both transformations behave very similarly and map the wave number structure of $E(\alpha, \omega)$ into similar spatial structures. In particular, if the horizontal coherence decreases smoothly with separation, the slant coherence must also decrease smoothly.

Figures $5 a$ and $5 b$ show the observed coherences $\gamma_{0 o}$ and $\gamma_{\ldots-}$ for a frequency in the internal wave continuum as a function of horizontal and slant separations. Only the horizontal coherence $\gamma_{00}$ decreases smoothly. All other coherences show a twoscale behavior: for separations less than a few meters they drop rapidly, and then for larger separations, they decrease similarly to $\gamma_{00}$ but at a lower level. The simplest model would ascribe the horizontal behavior of $\gamma_{00}$ to pure internal waves. The other three coherences must then be contaminated by noninternal wave fluctuations with smaller correlation scales. Pure instrumental noise can be excluded because it would produce the same drop in horizontal and slant coherences. More complicated models of the contamination have to be considered.

\section{Contamination by Temperature Fine Structure}

The vertical displacement has been estimated from temperature measurements. This procedure assumes a smooth mean temperature gradient. 'In the presence of temperature fine structure the estimate is contaminated by an additional signal which stems from the advection of layered temperature fine structure past the moored sensor by the internal wave field [cf. Phillips, 1971; Garrett and Munk, 1971; McKean, 1974]. This signal does not correspond to real vertical motion at the frequency considered. It leads to an increase of the displacement 


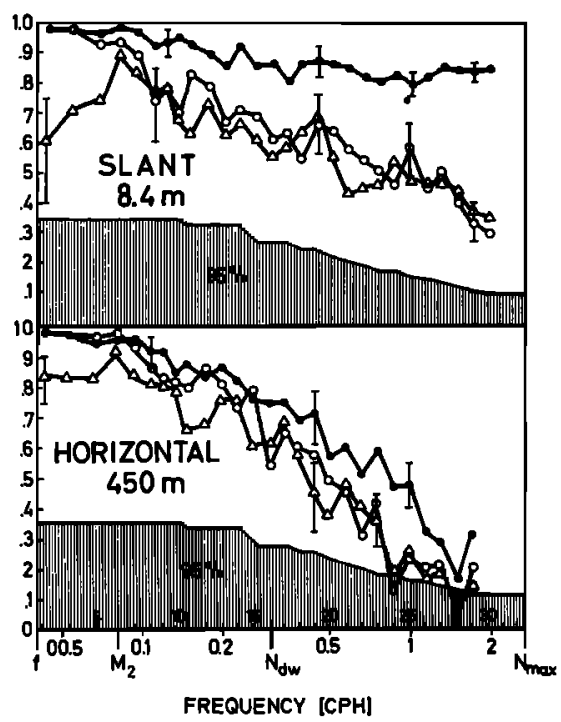

Fig. 4. Observed coherences for a slant and a horizontal separation. Full circle, $\gamma_{00}$; open circle, $\gamma_{--}$; triangle, $\gamma_{+++}$. Shaded area indicates $95 \%$ confidence limit for zero true coherence. Error bars are rms deviations.

energy and a decrease of the slant coherence. The horizontal coherence is not affected if the horizontal scale of the fine structure is larger than the array dimension.

Analyzing CTD and $\Delta T$ data from Iwex, which are more apt for fine structure investigations than temperature cross spectra, Joyce and Desaubies [1977] were able to confirm the contamination model of McKean [1974]. This model relates the relative energy $\delta_{f}$ (fine structure ratio) and the vertical coherence scale $a$ of the additional signal to characteristic parameters of the advected fine structure field and the internal wave field. The parameters $\delta_{f}$ and $a$ can also be estimated from Figure $5 b$. The rapid drop of $\gamma_{00}$ at small slant separations implies $a=O(2 \mathrm{~m})$. At larger separations the internal wave coherence $\gamma$ is reduced according to

$$
\gamma_{00}=\gamma / 1+\delta_{f}
$$

which yields $\delta_{f}=O(0.1)$. These values agree roughly with the findings of Joyce and Desaubies. Since we expect that the internal wave fluctuations are completely coherent over vertical separations of some meters, we may conclude from the increase of the coherence disparity for the slant coherence (Figure 4) that $\delta_{r}$ slightly increases with frequency from zero to about 0.2 . This also agrees with McKean's model.

\section{Contamination of Horizontal Current}

We have no well-established model at hand to explain the behavior of the coherences of the horizontal currents. An appropriate contamination model must satisfy various constraints. Both rotary components are affected in the same way, since the observed coherences $\gamma_{++}$and $\gamma_{--}$coincide (Figure 4) and the rotary energies $\boldsymbol{P}_{++}$and $\boldsymbol{P}_{--}$show no systematic deviation from the internal wave test (Figure $3 a$ ). Since both slant and horizontal coherences are affected, we have to exclude pure current fine structure, and since they are affected by a different amount, we have to exclude pure isotropic noise.

However, a combination of purely vertical fine structure and isotropic noise yields a satisfactory description. We choose this model because of its simplicity but have to bear in mind that contrary to the contamination of vertical displacement, there are no additional measurements to support this idea. The contamination could as well be caused by a single field with a more complicated correlation pattern (cf. paper 1). If we accept the partition into fine structure and noise, the energy and correlation scale parameters of this model can be determined from Figure 5. The relative noise energy $\eta_{n}$ is about 0.1 (Figure $5 b$ ), and the relative fine structure energy $\rho_{f}$ about 0.3 (Figure $5 a$; the total drop of the slant coherence is $\rho_{f}+\eta_{n}$ ). The relative energies increase slightly with frequency. The correlation scale of the noise must be less than the smallest separation for current meters $(\approx 7 \mathrm{~m})$. The current fine structure correlation drops vertically to zero also within $7 \mathrm{~m}$.

The fine structure signal in the horizontal currents may be due to advection of passive current fine structure past the sensors or caused by interaction of internal waves with the small-scale structure of the stratification [Eriksen, 1976]. Also, two-dimensional layered turbulence is conceivable. We cannot discriminate between these possibilities from our data set.

The noise field may be instrumental as well as three-dimensional isotropic turbulence confined to homogeneous layers, although both interpretations are not completely convincing. The nonwhite frequency dependence (se Figure 18 below) does not agree with simple conceptions of instrumental noise. Since the observed stratification during Iwex does not show completely homogeneous layers [Hayes et al., 1975], threedimensional turbulent motion should also be seen in the displacement data. If $d \theta_{L} / d z$ is the average temperature gradient within the layers, a turbulent field with relative energy $\eta_{n}$ should produce a temperature signal of relative energy

$$
\begin{aligned}
\delta_{f}=\frac{\omega^{2}+f^{2}}{\omega^{2}} \frac{N^{2}-\omega^{2}}{\omega^{2}-f^{2}}\left(\frac{d \theta_{L} / d z}{d T / d z}\right)^{2} \eta_{n} & \\
& \approx\left(\frac{N}{\omega} \cdot \frac{d \theta_{L} / d z}{d \bar{T} / d z}\right)^{2} \eta_{n}
\end{aligned}
$$

A typical value for the ratio of the temperature gradients is 0.3 [Hayes et al., 1975]. With $N / \omega \approx 5$ for the frequency considered in Figure 5 we find $\delta_{f} \approx 2 \eta_{n}=O(0.2)$. Both slant and horizontal coherenc of displacement should be decreased by this amount, which is not observed.

So far we have aligned our discussion of the contamination along coherences and have disregarded the energies. If we
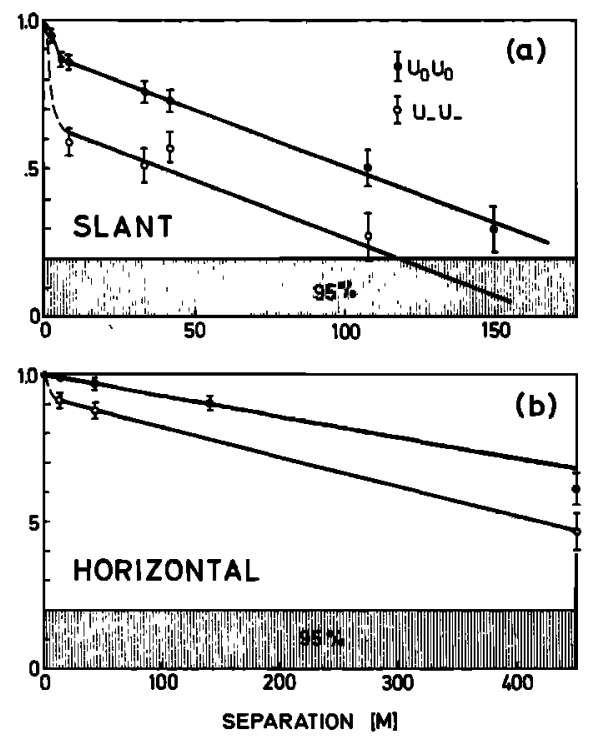

Fig. 5. Observed (a) slant and $(b)$ horizontal coherence for $\omega_{21} \stackrel{\neq}{=} .7$ hours versus separation. 


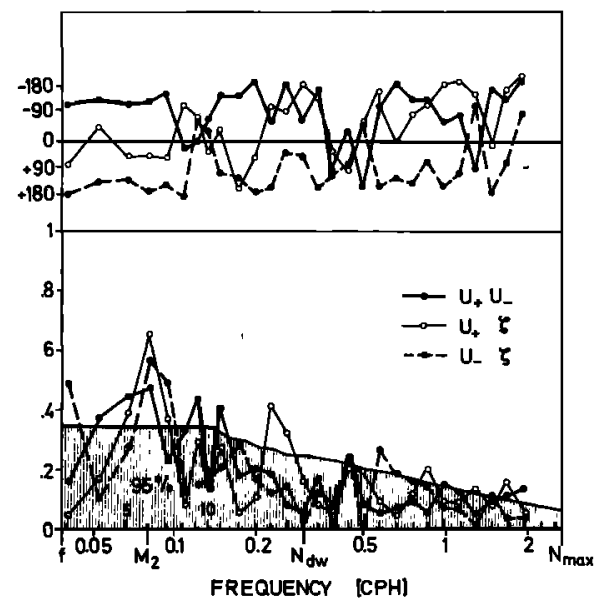

Fig. 6. Coherences and phases between different components of an instrument at $730-\mathrm{m}$ depth.

choose the parameters of the contamination fields such that the behavior of the coherences is well reproduced, the energies are incompatible. Considering the observed energy disparity and the estimated noise and fine structure energies, we end up with a 10-20\% excess of energy in the displacement. Since this occurs systematically at all frequencies, this excess is presumably not associated with a geophysical field but rather has to be explained by a systematic bias in the displacement spectra. These have been inferred from the temperature spectra by using the $\Delta T$ measurements, whereas the $N(z)$ profile used in the analysis has been calculated from separate CTD measurements. This is indeed the origin of the excess. As has been shown by T. M. Joyce (personal communication, 1976), who compared the CTD with the $\Delta T$ measurements, the CTD values for the buoyancy frequency are overestimated by about $10 \%$ (if the $\Delta T$ are correct).

For the above discussion we used a rather high frequency in the continuum. At lower frequencies where the main part of the energy is located the amount of contamination is small, and nearly all motions can be attributed to internal waves. We now come to a qualitative discussion of the internal wave spectrum, considering first isotropy and symmetry and then the distribution of energy in wave number space.

\section{Isotropy and Symmetry}

Horizontal isotropy of the fluctuations has already been demonstrated by Briscoe [1975], taking advantage of the special form of the array. He compared coherences of the three possible pairs of instruments in a horizontal level and found no dependence on the relative orientation. Also, the phases are zero, indicating that there is no preferred direction in the field.

There are other simple methods to test for isotropy which use only one-point measurements. For zero separation the coherences between different components vanish,

$$
\gamma_{+0}=\gamma_{-0}=\gamma_{+-}=0
$$

in case of isotropy (cf. equation (A38)). Notice that these conditions are necessary but not sufficient; e.g., if the field is symmetric but not isotropic we also have $\gamma_{+0}=\gamma_{-0}=0$. The ellipse stability $\gamma_{+-}{ }^{2}$ [Gonella, 1972] also vanishes for various anisotropic distributions, as was discussed in paper 1 . The tests (15) are not very sensitive. For the number of degrees of freedom of Iwex they allow only the rejection of isotropy for rather peaked distributions.

Figure 6 displays coherences and phases of an instrument at 730-m depth. In the internal wave continuum all three coherences are almost everywhere below the $95 \%$ confidence limit of true zero coherence. An isotropic distribution is here consistent with the data. At low frequencies, in particular at the semidiurnal tide $M_{2}$, the coherences peak significantly above the $95 \%$ confidence limit. Here isotropy must be rejected. The mean propagation direction of the tide can be estimated from the phase (cf. paper 1). In agreement with Noble and Joyce [1977], who used the same data, and Hendry [1975], who used Mode data, we find propagation toward the southeast. This may relate to the possible generation of baroclinic tides at the North American continental shelf. Notice, however, that our estimate of the propagation direction relies on the validity of the random internal wave description, which is quite unsatisfactory at $M_{2}$.

There are simple ways to decide whether or not the wave field may be regarded as symmetric. We mentioned already that $\gamma_{+0}=\gamma_{-0}=0$ for symmetry, but this holds also for isotropy. Tests which are not spoiled by isotropy involve the phases between the same components at vertically separated positions. For a symmetric wave field these phases vanish,

$$
\Phi_{++}=\Phi_{--}=\Phi_{00}=0
$$

whereas for asymmetric fields the sign of these phases is related to the vertical propagation direction. Figure 7 displays coherences $\gamma_{00}$ and phases $\Phi_{00}$ for two slant separations on one leg of the array. For the smaller separation the coherence is significantly above the $95 \%$ confidence limit, and the phase is zero within the error bars. For the larger separation the coherence is marginally significant, and the phase is different from zero at low frequencies. At higher frequencies the coherence is small, and the occasional peaks at intermediate frequencies occur at harmonics of the tide. This behavior points toward some slight asymmetries, which will show up more precisely when the complete data set is considered.

\section{Spectral Models of GM}

Since the wave field turned out to be essentially isotropic and symmetric, it is completely described by a spectrum $E(\alpha$, $\omega)$. We shall estimate its basic parameters. The analysis is

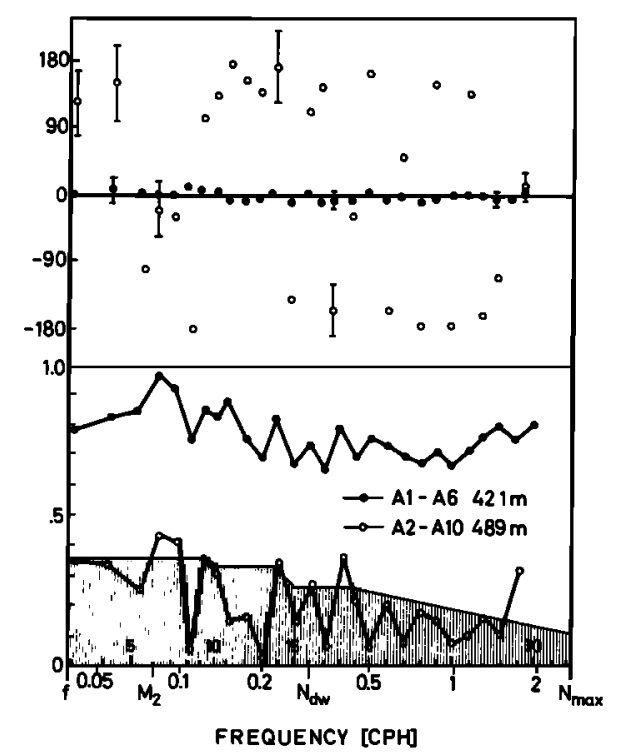

Fig. 7. Displacement coherences and phases for two slant separations on one leg of the mooring. 
TABLE 1. Parameter Values of the Wave Number Distribution (64) for the GM Models and the Model of Cairns and Williams [1976]

\begin{tabular}{lccc}
\hline & GM72 & GM75 & $\begin{array}{c}\text { Cairns and } \\
\text { Williams [1976] }\end{array}$ \\
\hline$j_{*}$ & 20 & 6 & 3 \\
$j_{e}$ & 20 & 11 & 9 \\
$j_{p}$ & 0 & 0 & 0 \\
$t$ & $\infty$ & 2.5 & 2 \\
$s$ & $\infty$ & 1 & 2 \\
$\Delta z_{1 / 2} \cdot \beta_{e}$ & 1.9 & 2.0 & 2.2 \\
\hline
\end{tabular}

Also given is the product of coherence scale and bandwidth.

oriented at Garrett and Munk's [1972, 1975] models (hereafter referred to as GM72 and GM75):

$$
E(\alpha, \omega)=E_{0} B(\omega) A\left(\frac{\alpha}{\alpha_{*}(\omega)}\right) \frac{1}{\alpha_{*}(\omega)}
$$

Here $E_{0}$ is the total energy, and

$$
B(\omega)=\frac{2 f}{\pi \omega}\left(\omega^{2}-f^{2}\right)^{-1 / 2}
$$

is the distribution in the frequency domain, which we have already discussed above. The normalized wave number distribution $A(\lambda)$ was modeled by a simple top hat in GM72 and by

$$
A(\lambda) \sim(1+\lambda)^{-t}
$$

in the more sophisticated GM75 model. A slightly different form was used by Cairns and Williams [1976]:

$$
A(\lambda) \sim\left(1+\lambda^{2}\right)^{-t / 2}
$$

The wave number scale $\alpha_{*}(\omega)$ was chosen as

$$
\alpha *(\omega)=j * \frac{\pi}{b N_{0}}\left(\omega^{2}-f^{2}\right)^{1 / 2}
$$

where $b N_{0}$ is the stratification parameter of an exponential profile $N(z)=N_{0} \exp (z / b)$. The parameter $j_{*}$ is the mode number scale associated with $\alpha_{*}$. This can be inferred from the WK B dispersion relation

$$
\alpha^{(J)}(\omega)=\frac{j \pi\left(\omega^{2}-f^{2}\right)^{1 / 2}}{\int_{-H}^{0} d z\left[N^{2}(z)-\omega^{2}\right]^{1 / 2}}
$$

A summary of the above models with values for the high wave number slope $t$ and the mode number scale $j *$ is given in Table 1.

\section{Bandwidth}

With reference to power spectral analysis we define the equivalent bandwidth $\alpha_{e}$ of a spectrum $E(\alpha, \omega)$ such that a box of width $\alpha_{e}$ and height $(E)=\int E^{2} d \alpha / \int E d \alpha$ has the same energy

$$
\alpha_{e}(\omega) \frac{\int d \alpha E^{2}(\alpha, \omega)}{\int d \alpha E(\alpha, \omega)}=\int d \alpha E(\alpha, \omega)
$$

This definition is particularly convenient if the moments of $E(\alpha, \omega)$ do not exist as, e.g., for the distributions (19) and (20). By using the dispersion relation the horizontal wave number bandwidth $\alpha_{e}$ can be converted to a mode number bandwidth $j_{e}$ or a local vertical wave number bandwidth

$$
\beta_{e}(\omega)=\alpha_{e}(\omega)\left(\frac{N^{2}-\omega^{2}}{\omega^{2}-f^{2}}\right)^{1 / 2} \approx j_{e} \frac{\pi N}{b N_{0}} \quad \omega<<N
$$

Values of $j_{e}$ for the above models can be found in Table 1. Notice that though the scales $j$. of the models differ greatly, the actually excited number of modes are quite similar.

The value of the bandwidth determines the scale $\Delta z_{1 / 2}$ where the vertical coherence as a function of separation drops to $\frac{1}{2}$. The qualitative relation $\beta_{e} \Delta z_{1 / 2}=O(1)$ can be stated more precisely by evaluating the coherence (12) for $r=0$. For a variety of spectral distributions we found (cf. paper 1)

$$
\beta_{e} \Delta z_{1 / 2} \approx 2
$$

with deviations of about $10 \%$. This is illustrated in Figure $8 a$ for distribution (20) with various values of $t$. This example also demonstrates that $\alpha_{e}$ is indeed a sensible definition of a bandwidth: spectra with the same equivalent bandwidth but different shapes produce a similar drop of coherence. On the contrary, for spectra with the same wave number scale $\alpha_{m}$ the coherence scale depends on the spectral shape (cf. Figure $8 b$ ). This can be stated more precisely. When $\alpha_{e}$ and $t$ are chosen as independent parameters, $t$ is a mere shape parameter. This is not true for the choice of $\alpha_{*}$ and $t$.

For estimation of $j_{e}$, consider the slant displacement coherences in Figure 9. Typical values of $\Delta z_{1 / 2}$ are $O(70-140 \mathrm{~m})$ for frequencies in the continuum and the inertial frequency. There is a lot of scatter, so that we may also expect some scatter in $j_{e}$. Particularly, the tide decreases much more slowly with $\Delta z_{1 / 2}=$ $O(500 \mathrm{~m})$. From (24) and (25), using $b N_{0}=0(5500 \mathrm{cph} \mathrm{m})$ for the Iwex profile and $N=2.5 \mathrm{cph}$, we obtain

$$
j_{e} \approx \frac{1400 \mathrm{~m}}{\Delta z_{1 / 2}}
$$

This yields $j_{e}=O(10-20)$ in the continuum and $j_{e}=O(3)$ at the tide. A similar analysis has been applied to horizontal coherences (cf. Figure 9) which leads to consistent results and thereby confirms the dispersion relation.

\section{Spectrum at High Wave Numbers}

Direct estimates of the wave number spectrum have been obtained from dropped and towed sensors. Hayes' [1975] yo-
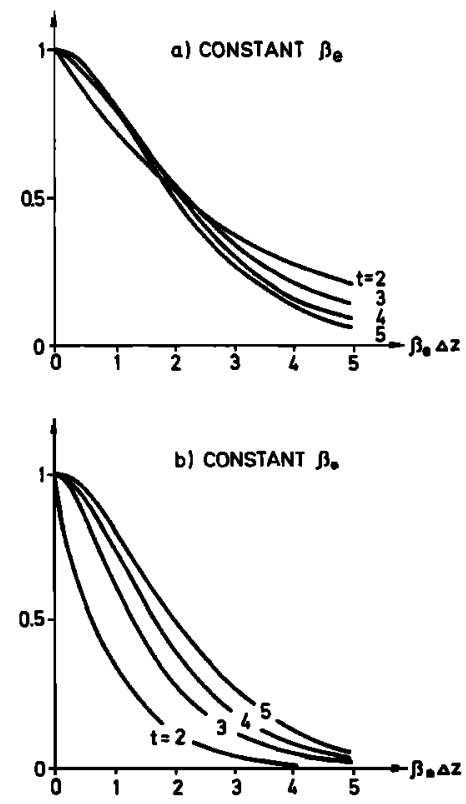

Fig. 8. Vertical coherence for internal wave spectra with wave number distribution $\sim\left(1+\lambda^{2}\right)^{-t / 2}$ for various values of $t,(a)$ for constant equivalent bandwidth and $(b)$ for constant scale parameter. 


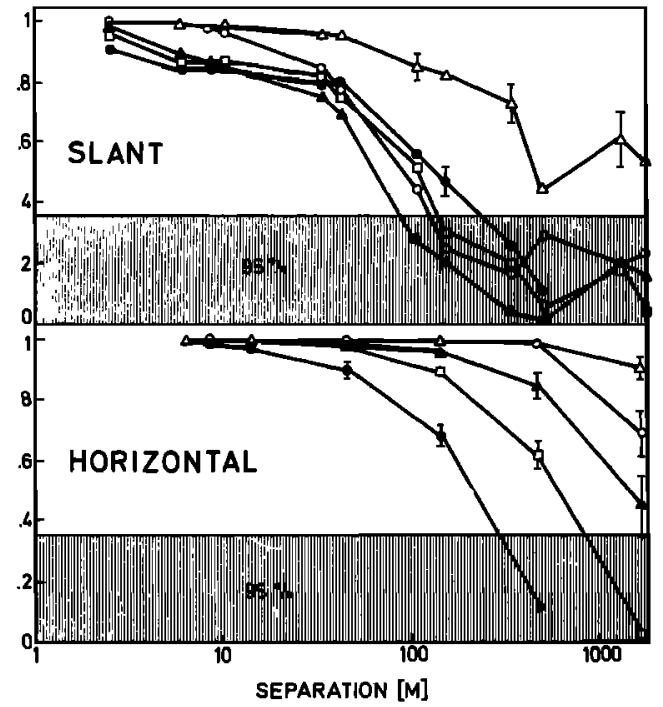

Fig. 9. Slant and horizontal displacement coherences for various frequencies versus separation. Open circle, $\omega_{3}$ (inertial); open triangle, $\omega_{6}$ (tidal); full triangle, $\omega_{13}\left(\hat{=} 5.0\right.$ hours); open square, $\omega_{20} \stackrel{\Leftrightarrow}{=} .0$ hours); full circle, $\omega_{28}(\stackrel{\cong}{=} 0.67$ hours $)$.

yo data, which were taken at the Iwex site, suggest a power law, $\beta^{-t}$, with $t \approx 2$ at vertical wavelengths larger than $50 \mathrm{~m}$. This agrees with the towed spectra $\sim \alpha^{-2}$ from the Mode area which have been measured by Katz [1975].

Moored coherences reflect the high wave number structure more indirectly. Cosine and Hankel transforms map the asymptotic behavior of the wave number distribution into the behavior of coherence at small separations. If the spectrum behaves as $\alpha^{-t}$ for $\alpha>\alpha_{0}$, then

$$
\gamma(r)=1-\text { const }\left(\alpha_{0} r\right)^{t-1}+O\left(\left(\alpha_{0} r\right)^{2}\right)
$$

for horizontal separations $r \ll 1 / \alpha_{0}$. An analogous relation holds for slant coherences. These relations can be utilized to estimate the high wave number slope $t$. We only use horizontal coherences, since the slant coherences are severely contaminated, was discussed above. Figure 10 displays $1-\gamma$ for the observed coherences versus separation. Obviously, the slope $t$ scatters between 2 and 3. More precise statements concerning a discrimination of Garrett and Munk's value of $t=2.5$ and Cairns and Williams' $t=2$ cannot be made.

To avoid infinite moments (e.g., rms shear), the wave number spectrum must have a cutoff, say, at $\alpha_{1} \gg \alpha_{0}$. Then (27) remains valid in the range $1 / \alpha_{1} \ll r \ll 1 / \alpha_{0}$, whereas the coherence drops as

$$
\gamma(r)=1-\text { const }\left(\alpha_{1} r\right)^{2}+O\left(\left(\alpha_{1} r\right)^{3}\right)
$$

for $r \ll 1 / \alpha_{1}$. From Figure 10 we may conclude $\alpha_{1}>O((10$ $\left.\mathrm{m})^{-1}\right)$. The smallest horizontal separation of the array $(r=2$ $\mathrm{m}$, not shown in the figure) would allow a more precise estimate of $\alpha_{1}$ only if the corresponding coherences were accurate to more than four digits, which is beyond instrumental accuracy.

\section{Spectrum at Low Wave Numbers}

The models of $A(\lambda)$ discussed so far have most of their energy at zero wave number and decrease monotonically. A cutoff at the lowest internal wave mode has been discussed by Garrett and Munk [1972]. It is conceivable that not the lowest but a higher mode is excited predominantly. Generally, peaks in the spectrum at nonzero wave numbers cause oscillations in the coherence as a function of separation which fade away with increasing separation. Evidence for such oscillations is occasionally found in the data set. Figure 11 shows the spatial behavior of the normalized displacement cospectrum for some typical frequencies.

Similar to the relation between bandwidth and coherence scale, there is a reciprocal relation between the peak wave number and the period of the oscillations. This may be used to locate the peak in the wave number domain. Explicitly, we find

$$
\beta_{p} \Delta z_{0} \approx \pi / 2
$$

where $\beta_{p}$ is the (vertical) peak wave number and $\Delta z_{0}$ is the vertical separation where the cospectrum crosses zero. In terms of the corresponding mode number, (29) becomes

$$
j_{p} \approx 1100 \mathrm{~m} / \Delta z_{0}
$$

The value of $\Delta z_{0}$ is very poorly determined but may be estimated at $O(500-1000 \mathrm{~m})$, which yields $j_{p}=O(1-2)$.

\section{Propagating Waves Versus Standing Modes}

So far we have interpreted our data in terms of vertically propagating waves. Most results remain valid if we use a modal interpretation. The vertical resolution of the array is not sufficient to decide whether the energy is confined to discrete dispersion curves or distributed continuously. However, the different statistical conceptions of the two representations imply certain differences which possibly can be detected in the data.

Consider again the vertical phases in Figure 7. For standing modes these phases must be zero, whereas they may be arbitrary for propagating waves. At low frequencies the phases differ significantly from zero. Here standing modes are an inadequate description. At higher frequencies the test is unable to discriminate between both representations. There are other phase relations which also differ. For standing modes we find

$$
\Phi_{+\zeta}+\Phi_{-\zeta}=0
$$

while for propagating waves

$$
\Phi_{+\zeta}+\Phi_{-\zeta}=\pi
$$

(Relation (31b) follows from (A33), and the modal relation analogously follows from (A41)). The phases $\Phi_{+\zeta}$ and $\Phi_{-\zeta}$ are shown in Figure 6 for an instrument at $730-m$ depth. At low frequencies, again, propagating waves are favored. In the continuum, phases are not well defined, since the corresponding

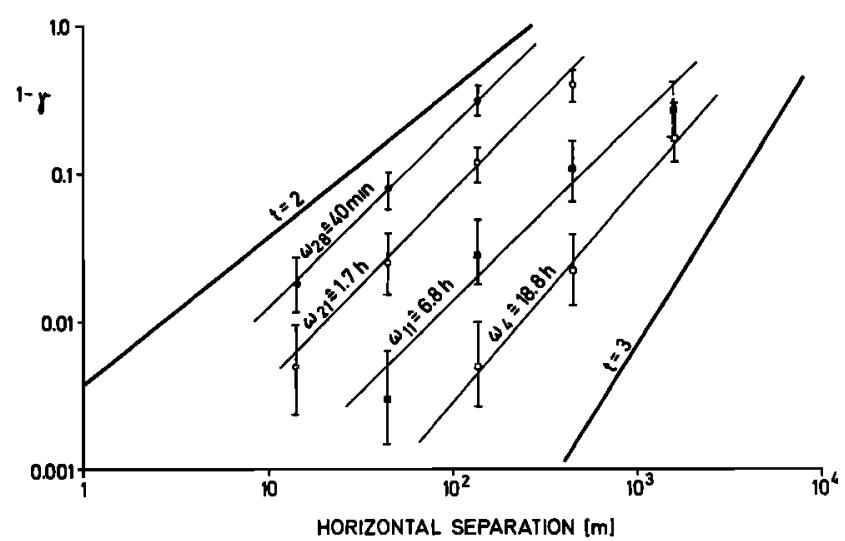

Fig. 10. Horizontal displacement coherence for four frequencies. Displayed is $1-\gamma_{00}$ versus horizontal separation. 


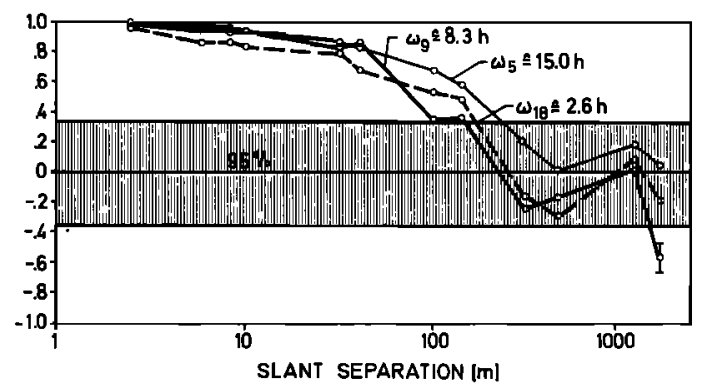

Fig. 11. Normalized displacement cospectrum for three frequencies versus slant frequency.

coherences are below the $95 \%$ confidence level. Here no discrimination is possible.

These phase tests are unable to distinguish between standing modes and a symmetric field of propagating waves. This must be attributed to the fact that our cross spectra are strongly time averaged, which because of ergodicity corresponds to a space average. Vertical average destroys information about the fixed phase relationship of standing modes.

Nevertheless, we expect that the propagating wave representation breaks down at high frequencies in two ways. First, the WKB approximation of the vertical eigenfunctions is not applicable in the proximity of the turning point. More refined methods, e.g., the Airy function approximation, should be used [Desaubies, 1973]. Second, the statistical concept of propagating waves breaks down because approaching the turning level upward and downward propagating waves have increasingly deterministic phase relations due to reflection at that level. This" phase locking leads to a hump in autospectra and an increase of the same component coherences near the local buoyancy frequency [Desaubies, 1975]. This increase is indeed observed in the displacement data (e.g., Figure 7), while it is obscured in the current data due to strong contamination. This demonstrates that at the very high frequencies, standing modes are the preferable description.

In summary, propagating waves dominate at low frequencies, and standing modes at high frequencies, whereas both descriptions are adequate at intermediate frequencies.

\section{Rigorous Analysis}

So far we have examined a selected part of the Iwex data and inferred some important properties of the corresponding field of motion in a qualitative manner. Although fairly detailed results could be obtained, the approach has two shortcomings. Since we have only considered a rather small percentage $(\approx 5 \%)$ of all data, we may have overlooked additional or even contradicting information. Furthermore, it is desirable to get quantitative results which reduce the information in the data set to a manageably small set of parameters. To overcome these defects and to arrive at a consistent and concise interpretation of all data, it is necessary to generalize the above analysis so that all data are involved in a systematic way. Here we discuss the basic concepts of such an analysis; a short summary can be found in the paper by Olbers et al. [1976].

\section{Likelihood Ratio Test}

For each frequency, all measured (complex) cross spectra $A_{\mu \nu}{ }^{j}(\omega)$ will be represented by the (real) column vector

$$
\mathbf{y}=\left\{y_{l} ; l=1, \cdots, L\right\}=\left\{A_{\mu \nu}{ }^{\prime}{ }^{\prime}(\omega) ; \text { all } i, j, \mu, \nu\right\}
$$

It is useful to think of $y$ as the data point in the $L$-dimensional cross-spectral space (cf. Figure 12). For most frequencies in this experiment, $L=1444$.

Time series of finite length only provide estimates of the cross spectra. Hence the data point $y$ has to be regarded as the realization of a random variable. The covariance matrix

$$
\boldsymbol{S}_{l^{\prime}}=\operatorname{Cov}\left[y_{l}, y_{l^{\prime}}\right]
$$

can be estimated by using (5). The joint probability density function is nearly Gaussian because of the high number of degrees of freedom.

Relations like (7) predict model values of the cross-spectral matrix which we represent by a model point $\hat{y}=\left\{y_{l} ; l=1, \cdots\right.$, $L\}$ in the cross-spectral space (cf. Figure 12). Obviously, we regard the model $\hat{y}$ as consistent with the data $y$ if both points coincide within the confidence limits. A precise formulation of this criterion is found from the maximum likelihood principle, which yields as appropriate statistic

$$
\epsilon^{2}=(y-\hat{y})^{+} \mathbf{W}(\mathbf{y}-\hat{y})
$$

with the weight matrix $\mathbf{W}=\mathbf{S}^{-1}$. If $\mathbf{W}$ is interpreted as a metric in the cross-spectral space, then $\epsilon$ is the distance between model and data point.

The statistic $\epsilon^{2}$ follows a normalized $\chi^{2}$ distribution with $L$ degrees of freedom. For a given probability level (always $95 \%$ throughout this paper) we reject the model if $\epsilon^{2}$ exceeds the 0.05 percentile $\chi_{L, 0.05}{ }^{2}$ of the $\chi^{2}$ distribution [Abramowitz and Stegun, 1972]. If

$$
\epsilon^{2}<\chi_{L, 005}^{2}
$$

we accept the model as consistent, being aware that alternative models are not precluded.

To perform the likelihood ratio test, the data covariance matrix $\mathbf{S}$ has to be inverted. Regardless of practical difficulties arising from its size $(L \times L)$, this is only possible if there are no redundant data, since otherwise $\mathbf{S}$ would be singular. This certainly occurs in the present experiment, where many data (e.g., from sensors with small separation) are strongly correlated. Therefore the weight matrix $\mathbf{W}$ must be modified, as will be discussed later.

A consistent model will be constructed in two steps. In the first step (consistency tests) we will test some basic properties of the field of motion. In the second step (inverse method) we will determine the characteristic parameters of the observed fluctuations.

\section{Consistency Tests}

It has already been mentioned that for the internal wave model (7) there exist relations among the different components

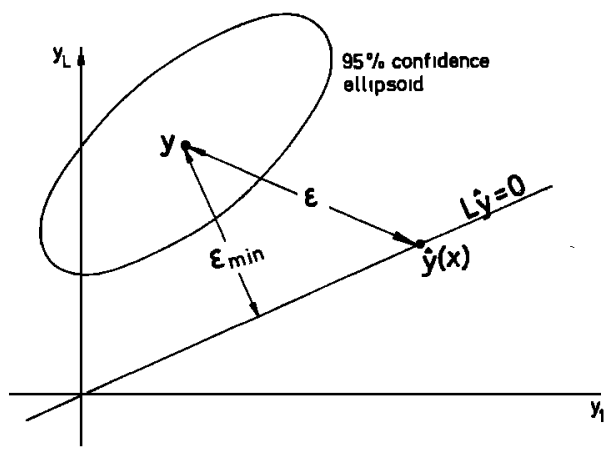

Fig. 12. Schematic graph of data and model point in cross-spectral space. 
of the cross-spectral matrix, e.g.

$$
(\omega+f)^{2} P_{++}(\omega)-(\omega-f)^{2} P_{--}(\omega)=0
$$

which hold for arbitrary energy spectra. As has been demonstrated, such consistency relations can be utilized to test whether or not the observed fluctuations can be attributed to internal waves.

Consistency relations can formally be written as

$$
\sum_{l} L_{r l} \hat{y}_{l}=0 \quad r=1, \cdots, R
$$

Here the index $r$ counts the number of (linearly independent) relations. For internal wave model classes, complete sets of these consistency relations are listed in the appendix (see also Müller and Siedler [1976]); other model classes will be introduced below. In the cross-spectral space any specific model point lies in the ( $L-R)$-dimensional hyperplane defined by (37) (see Figure 12). This restriction may be quite strong; e.g., an isotropic and symmetric field of propagating internal waves must satisfy $R=854$ relations among $L=1444$ cross spectra.

To decide whether or not a specific model class is consistent with the observations, we obviously have to consider the minimal distance $\epsilon_{\mathrm{min}}$ between the hyperplane $\mathrm{L} \hat{y}=0$ and the data point $y$. By straightforward algebra this is found to be

$$
\epsilon_{\mathrm{min}}{ }^{2}=(\mathrm{Ly})^{+} \mathrm{VLy}
$$

where $\mathrm{V}=\left(\mathrm{LSL}^{+}\right)^{-1}$. The (squared) distance $\epsilon_{\mathrm{m} / \mathrm{n}}{ }^{2}$ again follows a $\chi^{2}$ distribution but in contrast to $\epsilon^{2}$ with $R$ rather than $L$ degrees of freedom, since only $R$ cross spectra are constrained by the consistency relations.

Again, the matrix

$$
\mathbf{T}=\mathrm{LSL}^{+}
$$

may be expected to be singular. We therefore approximate $\mathrm{V}$ as

$$
V_{r r^{\prime}} \approx \delta_{r r^{\prime}} / T_{r r}
$$

and use the normalized statistic

$$
\Delta^{2}=\frac{1}{R} \sum_{r}(\mathrm{Ly})_{r} / T_{r r} \approx \frac{1}{R} \epsilon_{\min }^{2}
$$

which follows a $\chi^{2}$ distribution with expectation value $\left\langle\Delta^{2}\right\rangle=1$ and

$$
R_{\text {eff }}=R^{2}\left\{\sum_{r r^{\prime}} T_{r r^{\prime}} /\left(T_{r r} T_{r^{\prime} r^{\prime}}\right)\right\}^{-1}
$$

degrees of freedom. It follows that $R_{\text {erf }} \leq R$. For Iwex we find $R_{\text {eft }} \approx 0.1 R$ within a factor of 2 .

The calculation of the statistic $\Delta^{2}$ is straightforward. The results for various model classes are displayed in Figure 13 as a function of frequency. The model class and the number $R$ of consistency relations per frequency are given in the inlet of the figures. Also shown is the $95 \%$ confidence limit (dashed line). For a single frequency we reject the model class if the calculated value of $\Delta^{2}$ is larger than the $95 \%$ confidence limit. For a frequency band we also reject it if the values of $\Delta^{2}$ do not scatter randomly about the expectation value $\left(\Delta^{2}\right)=1$.

The first tests consider some simple model classes.

Zero model (Figure 13a). The simplest hypothesis is that all cross spectra vanish,

$$
A_{\mu \nu}{ }^{l j}=0 \quad \forall i, j, \mu, \nu
$$

which implies a coefficient matrix $L_{r l}=\delta_{r l}$. The statistic is explicitly given by

$$
\Delta^{2}=\frac{1}{L} \sum_{i, f, \mu, \nu}\left(A_{\mu \nu}{ }^{i j}\right)^{2} / \operatorname{Var}\left(A_{\mu \nu}{ }^{l j}\right)
$$

The model class must clearly be rejected at all frequencies. The strong increase of $\Delta^{2}$ with frequency reflects the increasing accuracy of the observed cross spectra because $\operatorname{Var}\left(\mathrm{A}_{\mu \nu}{ }^{l j}\right) \sim$ $\nu^{-1}$ according to (5). To demonstrate this dependence, the equivalent number $\nu$ of degrees of freedom is also shown in Figure $13 a$.

White noise (Figure 13a). Here white noise is defined as a field for which all cross spectra vanish, with the exception of the autospectra, which can take arbitrary values, i.e.,

$$
A_{\mu \nu}{ }^{i j}=0 \quad \mu \neq \nu \text { or } i \neq j
$$

This model class must also be rejected at all frequencies.

Fine structure (Figure 13a). We consider the model class for which all cross spectra from vertically separated instruments vanish,

$$
A_{\mu \nu}{ }^{l j}=0 \quad z^{i} \neq z^{J}
$$

whereas the remaining cross spectra may take arbitrary values. This model class reflects the principal features of density and current fine structure in the ocean, which has a small vertical and large horizontal coherence scale. In our definition the vertical coherence scale is assumed to be smaller than the smallest vertical separation in the array. The horizontal scale and the energies remain unspecified. This fine structure model must also be rejected at all frequencies.

None of these simple model classes can be accepted. This is not surprising, but we are assured that consistency tests indeed sort out unreasonable model classes, especially because the largest values for $\Delta^{2}$ are found for the most unreasonable model, the zero model. Furthermore, the more accurate the data, the better the tests discriminate between different model classes. It will hence be more difficult to find a consistent model at high than at low frequencies.

The next group tests some basic properties of existing internal wave models.

GM model class (Figure 13b). Though Garrett and Munk start from vertically standing modes, they take locally defined vertical averages and smear out the modal structure into a continuum in wave number-frequency space. As was mentioned above, this procedure destroys the concept of standing modes. The basic assumptions of the GM model are then as follows:

1. The observed fluctuations are a realization of a statistically stationary process.

2. The fluctuations are horizontally homogeneous.

3. The fluctuations are a superposition of free linearly propagating internal waves.

4. The wave field scales vertically according to WKB theory.

5. The wave field is horizontally isotropic.

6. The wave field is vertically symmetric.

The last assumption is a consequence of Garrett and Munk's modal approach. The approximate stationarity has been proven by C. Frankignoul and T. M. Joyce (1977); here we test all linear relations among the cross spectra which follow from the other assumptions. These relations are derived in the appendix and will be discussed in some detail later. Figure $13 b$ demonstrates that the GM model class cannot be accepted. Strong rejection occurs at high frequencies and tide. 

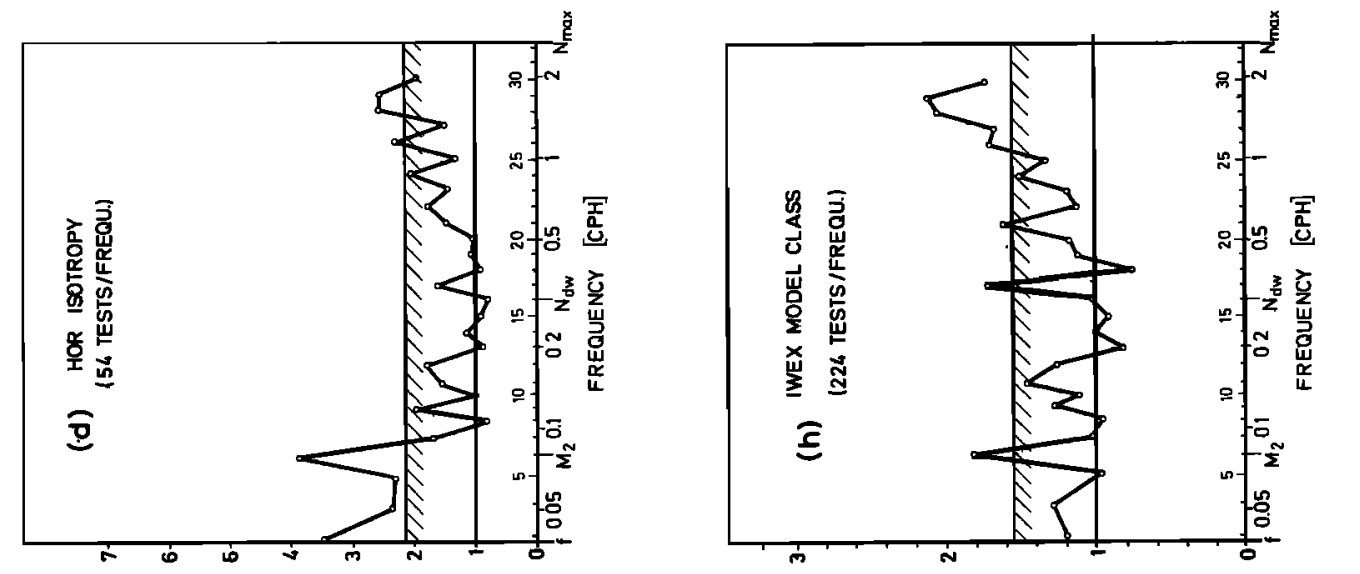

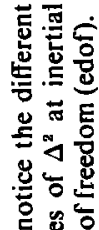

远
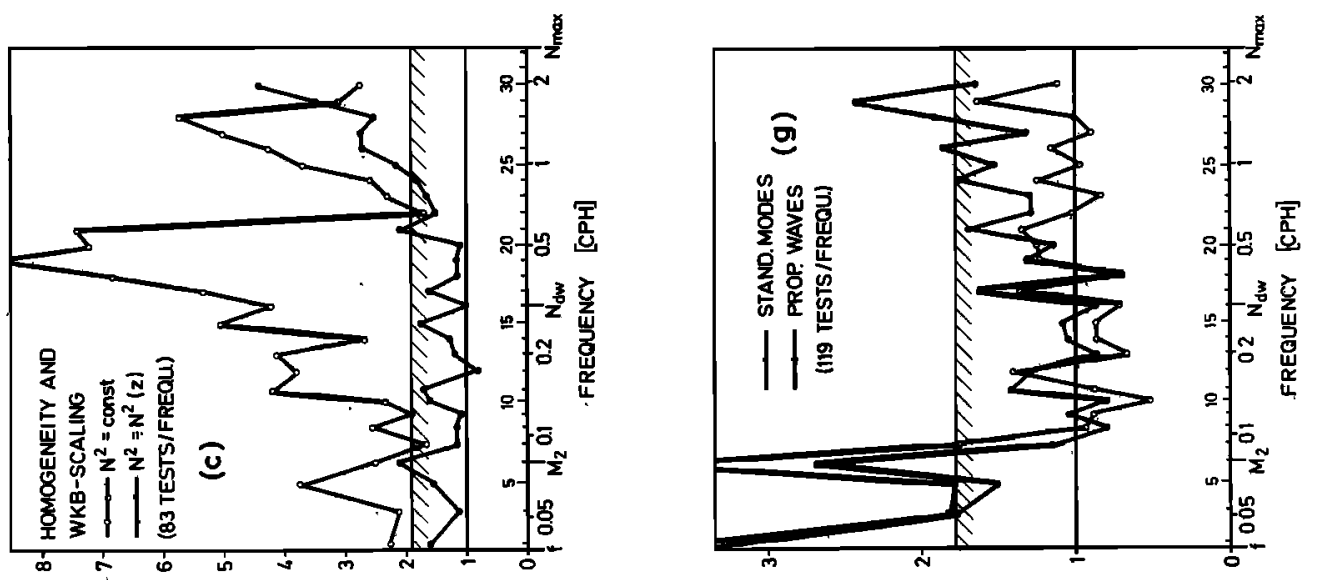

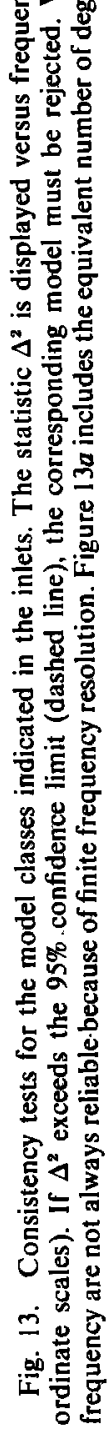
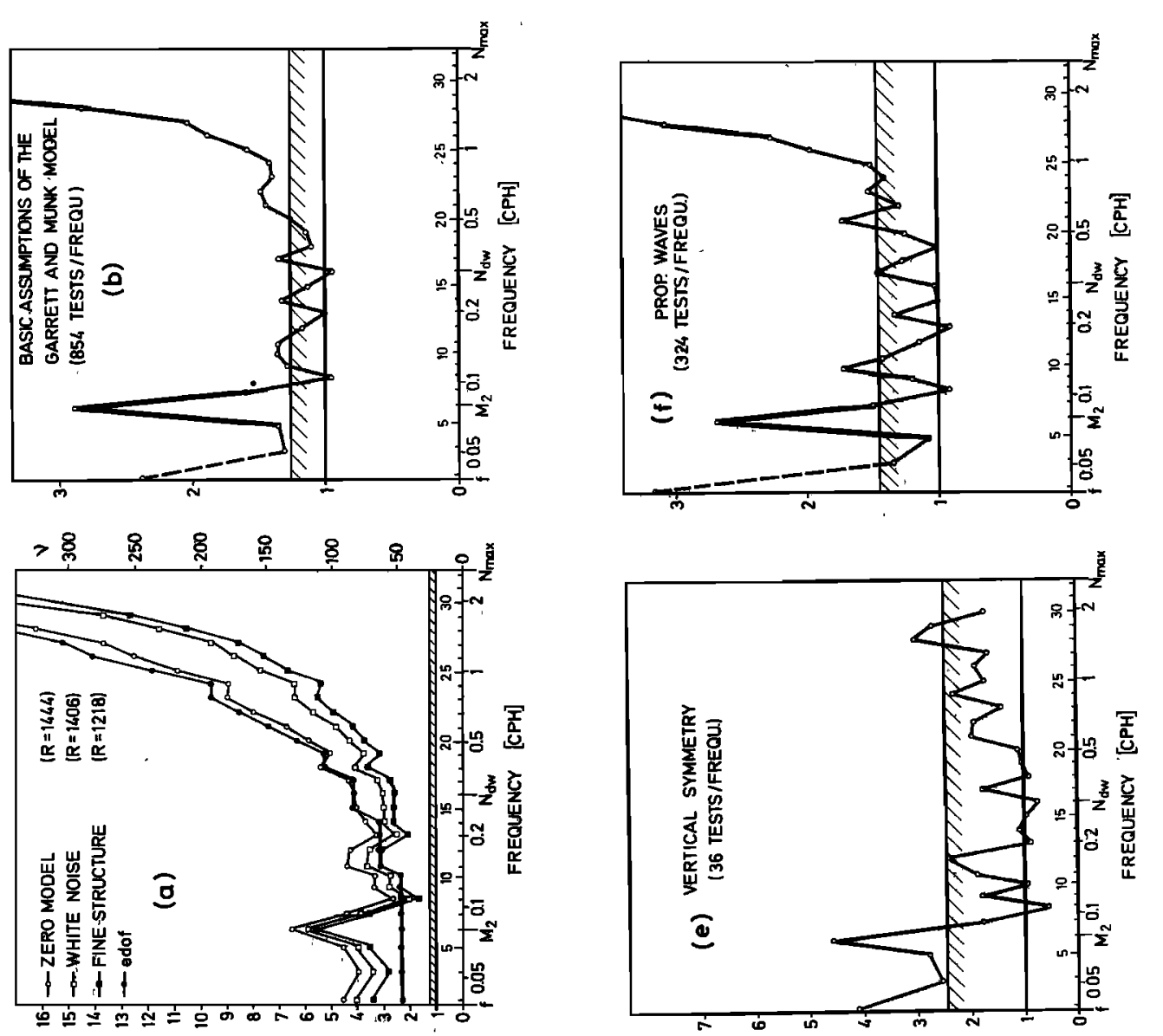
The model must also be rejected at medium frequencies, since the values for $\Delta^{2}$ do not scatter randomly about the expectations value $\left\langle\Delta^{2}\right\rangle=1$, indicating slight but systematic inconsistencies. The estimate at the inertial frequency is not reliable because of finite frequency resolution. The following tests investigate which of the basic assumptions 2-6 are violated most.

Homogeneity and WKB scaling (Figure 13c). WKB requires that cross spectra from different instruments satisfy the scaling relations

$$
A_{\mu \nu}{ }^{u} / D_{\mu \nu}\left(z^{l}\right)-A_{\mu \nu}{ }^{j} / D_{\mu \nu}\left(z^{j}\right)=0
$$

where

with

$$
\Omega(z)=\left(\frac{N^{2}(z)-\omega^{2}}{\omega^{2}-f^{2}}\right)^{1 / 2}=\frac{\beta(z)}{\alpha}
$$

The relations for horizontal homogeneity are included for $z^{\imath}=$ $z^{\prime}$. Relations (47) are violated for the highest frequencies. The distribution of $\Delta^{2}$ for the remaining frequencies only indicates slight inconsistencies. The violation at high frequencies is presumably due to the proximity of the turning point where the WKB solution becomes singular. This explanation is confirmed by the drop of $\Delta^{2}$ at the twenty-second frequency, since for higher frequencies the deepest three instruments are excluded from the analysis. To demonstrate the sensitivity of the test, the figure also displays $\Delta^{2}$ for a constant buoyancy profile, or equivalently, for a vertically homogeneous field. Such a model is obviously inadequate. Notice again the pronounced drop at the twenty-second frequency.

Horizontal isotropy (Figure (3d). For an isotropic field the cross spectra do not change when rotated about the vertical axis, implying the isotropy relations

$$
A_{+0}{ }^{i i}=A_{-0}{ }^{i l}=A_{+-}{ }^{i i}=0
$$

Because of the special array geometry there are also isotropy relations among cross spectra from horizontally separated instruments such as the independence of coherences on the orientation [Briscoe, 1975]. These are excluded here. For internal waves there exist additional isotropy relations (cf. appendix) which have been considered in the test of the GM model class but are also excluded here. The assumption of isotropy can be accepted at medium frequencies. At high frequencies we find a slight anisotropy, and at low frequencies a strong one.

Vertical symmetry (Figure 13e). For a symmetric field the cross spectra do not change when reflected at a horizontal plane, implying the symmetry relations

$$
A_{+0}{ }^{i l}=A_{-0}{ }^{i l}=0
$$

The result of this test is similar to the one above, partly because the symmetry relations form a subset of the isotropy relations.

Propagating waves (Figure 13f). The preceding tests show that the violation of the GM model class cannot primarily be ascribed to failing of homogeneity, scaling, isotropy, or symmetry relations. Hence the fluctuations are not propagating internal waves. This is confirmed when the consistency relations

$$
A_{++}{ }^{\jmath}+A_{--}{ }^{l}-\frac{\omega^{2}+f^{2}}{\omega^{2}} \Omega\left(z^{l}\right) \Omega\left(z^{J}\right) A_{00} l \jmath=0
$$

$$
\begin{aligned}
(\omega+f)^{2} A_{++}{ }^{l j}-(\omega-f)^{2} A_{--}{ }^{l j} & =0 \\
\Omega\left(z^{j}\right)(\omega+f) A_{+0}{ }^{i j}-\Omega\left(z^{i}\right)(\omega-f) A_{0-}{ }^{l \jmath} & =0 \\
\Omega\left(z^{j}\right)(\omega-f) A_{-0}{ }^{l \jmath}-\Omega\left(z^{i}\right)(\omega+f) A_{0+}{ }^{l j} & =0
\end{aligned}
$$

for propagating internal waves are tested.

Relation (52a) compares the vertical displacement with the horizontal current field. For zero separation it implies that the vertical kinetic or potential energy is a fixed fraction of the horizontal kinetic energy (cf. equation (9)). For nonzero separation it implies (together with $(52 b)$ ) that coherence and phase of displacement and current are equal (cf. equation (11)). Deviations from (52a) express the energy and coherence disparities discussed above. Relation (52b) compares the clockwise and anticlockwise parts of the current field, and $(52 c)$ and $(52 d)$ the cross spectra between displacement and current. Relations $(52 a-52 d)$ are strongly violated for high frequencies and the tidal frequency and slightly violated for medium frequencies. A detailed analysis reveals that $(52 a)$ is violated most of all, implying that the rejection of propagating waves must primarily be attributed to the energy and coherence disparities. Can these inconsistencies be removed by choosing standing modes?

Standing modes (Figure $13 \mathrm{~g}$ ). These satisfy relation $(52 b)$ between the horizontal current components. There is no relation between current and displacement which is equivalent to (52a), and there are also no relations involving cross spectra between current and displacement at vertically separated instruments. For purely horizontal separation, standing modes satisfy

$$
\begin{gathered}
(\omega+f) A_{+0}{ }^{i \jmath}+(\omega-f) A_{0-}{ }^{i \jmath}=0 \\
(\omega-f) A_{-0}{ }^{i \jmath}+(\omega+f) A_{0+}{ }^{i \jmath}=0
\end{gathered}
$$

which differ from relations $(52 c)$ and $(52 d)$ and thus provide a tool to discriminate between standing modes and propagating waves. Figure $13 g$ displays the statistic $\Delta^{2}$ obtained from all consistency relations for standing modes together with the corresponding ones for propagating waves. Both descriptions are clearly invalid at the tidal frequency, propagating waves being slightly better. Relation $(52 b)$, which applies to both descriptions, contributes most to the inconsistency at $M_{2}$ because of the strong anticlockwise rotation of the barotropic tidal current. As has already been discussed, both descriptions are equivalent at medium frequencies. At high frequencies, standing modes provide a more adequate model class, though propagating waves are not strongly excluded. However, standing modes cannot remove the energy and coherence disparities. If high or many modes are excited, a vertically averaged field of standing modes must satisfy $(52 a)$ as well. If only a few modes are excited, there might be a systematic violation of $(52 a)$, but a few modes are not consistent with the observed coherence scale.

Iwex model class (Figure 13h). None of the internal wave model classes gives a consistent description because none of them could explain the energy and coherence disparities. The analysis in the first part of this paper suggests a model class consisting of propagating internal waves contaminated by current and temperature fine structure and by current noise. This more general model only satisfies a subset of the consistency relations $(52 a-52 d)$ for propagating waves. In the presence of current noise (with the same amount in the clockwise and anticlockwise components), (52a) and (52b) need not be satisfied for zero separations. Adding current and temperature fine structure with an arbitrary horizontal coherence scale, $(52 a)$ 
and $(52 b)$ need not be satisfied for horizontal separations. Considering furthermore that the CTD measurements might provide a biased Brunt-Väisälä profile, $(52 a)$ need not be satisfied for arbitrary separations. The Iwex model class is hence characterized by relations $(52 c)$ and $(52 d)$ for arbitrary separations and $(52 b)$ for slant separations. Generally, this model class seems to provide a consistent description, though it must be remembered that the test uses only part of the data set. The addition of noise and fine structure cannot remove inconsistencies at high frequencies. This reflects the inadequacy of the WKB solutions in the proximity of the turning point, which might be removed by using Airy [Desaubies, 1975] or standing mode solutions. Both account for phase locking near the turning point. We will use the propagating wave model, since it is conceptually and computationally simpler.

\section{Least Squares Fit}

We have demonstrated in the previous section that the Iwex model class, in contrast to pure internal wave models, might lead to a consistent interpretation of the data. Suppose we parameterize this model in terms of a number $P$ of unknown parameters $\mathbf{x}=\left(x_{1}, \cdots, x_{p}\right)$ so that we have a model $\hat{\mathbf{y}}=\hat{\mathbf{y}}(\mathbf{x})$. Since the number $L$ of data is typically $O\left(10^{3}\right)$, whereas $P$ will be $O(20)$, the system is overconstrained, meaning that the equation $y=\hat{y}(x)$, or equivalently $\epsilon^{2}(x)=0$, has no solution for $x$. However, since we reject a model point $\hat{y}$ only if its distance from the data point $y$ exceeds a certain critical value, it is sensible to determine $\mathbf{x}$ by requiring

$$
\epsilon^{2}(x)=(y-\hat{y}(x))^{+} \mathbf{W}(y-\hat{y}(x))=\min
$$

An application of this generalized least squares principle to internal wave problems has been given by Schott and Willebrand [1973].

The solution to (53) is constructed in two steps: (1) A zeroorder estimate $x_{0}$ is obtained by using the information of the preliminary analysis and a simple numerical minimum technique. (2) This estimate is then improved by linearizing the model $\hat{y}(\mathbf{x})$ at $\mathbf{x}=\boldsymbol{x}_{\mathbf{0}}$

$$
\hat{y}(x)=\hat{y}\left(x_{0}\right)+\mathbf{A}\left(x-x_{0}\right)
$$

where

$$
\begin{aligned}
A_{l \alpha}= & \left(\partial \hat{y}_{l} / \partial x_{\alpha}\right)_{\mathbf{z}=\mathbf{x}_{0}} \\
& l=1, \cdots, L \quad \alpha=1, \cdots, P
\end{aligned}
$$

Condition (53) then yields the normal equations

$$
\mathbf{M}\left(\mathrm{x}-\mathrm{x}_{0}\right)=\mathbf{A}^{+} \mathbf{W}\left[\mathbf{y}-\mathbf{y}\left(\mathbf{x}_{0}\right)\right]
$$

with

$$
\mathbf{M}=\mathbf{A}^{+} \mathbf{W A}
$$

By inversion of the $P \times P$ matrix $\mathrm{M}$, a new value $\mathrm{x}$ is obtained, and step 2 may iteratively be repeated.

It has been noted by Jackson [1972] that even in the overconstrained case $(L>P)$ problem (53) can be underdetermined, since $\mathbf{M}$ may be singular. This case will occur if some parameters or parameter combinations essentially describe the same features in the data and therefore are dependent. For solving (53), $\mathbf{M}^{-1}$ has then to be interpreted as the generalized (Moore-Penrose) inverse of $\mathbf{M}$ (for details see paper 1).

Since the parameters $x$ are determined from the random variables $y$, they are also random quantities. Their covariance matrix $R_{\alpha \beta}=\operatorname{Cov}\left(x_{\alpha}, x_{\beta}\right)$ is calculated from (56):

$$
\mathbf{R}=\mathbf{M}^{-1} \mathbf{A}^{+} \mathbf{W} \text { SWAM }^{-1}
$$

This matrix gives two kinds of information. The diagonal elements determine the error bars for the parameters and therefore reflect the accuracy of the model. To some degree this accuracy can be controlled by changing the number of parameters. Adding more parameters generally decreases the accuracy of the parameters already present. This tradeoff between resolution and variance is quite similar to the situation in spectral analysis. For a thorough discussion, see Jackson [1972].

The off-diagonal elements of $\mathbf{R}$ determine the parameter correlation. Highly correlated parameters describe the same data features. Such a parameterization of a model does not affect the result of the fit but may prevent a unique physical interpretation of the parameters. Formally, one can construct a set of statistically independent parameters by diagonalizing $\mathbf{R}$. For nonlinear models the new parameters generally do not possess clear physical meaning.

\section{Weight Matrix}

The information which is extracted from the data set depends on the choice of the metric $W$. A quantitative measure of this information is given by the 'effective' number of data

$$
L_{\text {efr }}=\frac{2\left\langle\epsilon^{2}\right\rangle^{2}}{\operatorname{Var}\left(\epsilon^{2}\right)}=\frac{(\operatorname{Tr} S W)^{2}}{\operatorname{Tr} S W S W}
$$

The maximum likelihood principle maximizes this number. If $\mathbf{S}$ is nonsingular, this principle leads to $\mathbf{W}=\mathbf{S}^{-1}$ and $L_{\mathrm{efr}}=L$. If $\mathbf{S}$ is singular, $\mathbf{W}$ is given by the generalized inverse of $\mathbf{S}$, and $L_{\text {err }}<L$. Then $L_{\text {err }}$ is the number of independent data. The interpretation of $L_{\mathrm{err}}$ as a measure of information is valid only in a restricted statistical sense. Nonstatistical information, e.g., positions or separations associated with the data, are not included in this measure.

The construction of the generalized inverse of $\mathbf{S}$ meets with considerable computational problems because of its large size. We hence restrict ourselves to diagonal metrics $W_{l l^{\prime}}=w_{l} \delta_{l l}$ and require by

$$
\sum_{l l^{\prime}}\left(S_{l l^{\prime}} w_{l^{\prime}}-\delta_{l l^{\prime}}\right)^{2} \frac{S_{l^{\prime} l^{\prime}}}{S_{l l}}=\min
$$

that $\mathbf{W}$ should closely resemble the structure of the maximum likelihood weight. Condition (60) leads to the weight

$$
w_{l}=\frac{1}{S_{l l}}\left(\sum_{l^{\prime}} \frac{S_{l l^{\prime}}^{2}}{S_{l l} S_{l^{\prime} l^{\prime}}}\right)^{-1}
$$

which scales the data by their rms standard deviations and weights strongly correlated data less than uncorrelated data. This is reasonable since the latter, in a statistical sense, do contribute more information. The effective number of data calculated with the weights (61) is shown in Figure 14. While this weight yields a number which is larger by a factor of 2 compared to the usually employed weights $w_{l}=1 / S_{l l}$, it is still only about $10 \%$ of the total data number. This ratio demonstrates the overall coherence of the Iwex data set. Only about 100 data provide statistically independent information to determine the $O(20)$ parameters of the model.

\section{The Iwex Spectral Model}

Guided by the physical arguments of the preliminary analysis and assured by the consistency test, we represent the ob- 


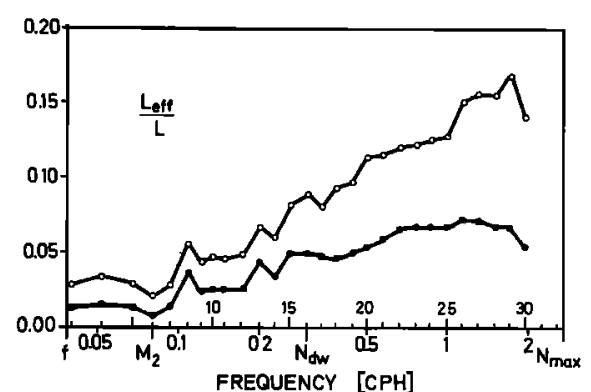

Fig. 14. Ratio $L_{\text {err }} / L$ of effective to total number of data for two weights. Open circle, weights used for Iwex; full circle, weights $1 / S_{l l}$.

served cross spectra by the model

$$
\begin{aligned}
& \hat{A}_{\mu \nu}{ }^{i j}=I_{\mu \nu}{ }^{i j}+F_{\mu \nu}{ }^{i j}+N_{\mu \nu}{ }^{j j} \quad \mu, \nu=+,- \\
& \hat{A}_{00}{ }^{j}=I_{00}{ }^{i j}+F_{00}{ }^{i j} \\
& \hat{A}_{\mu_{0}}{ }^{l j}=I_{\mu_{0}}{ }^{1 j} \quad \mu=+,- \\
& \hat{A}_{0 \mu}{ }^{i j}=I_{0 \mu}{ }^{l j} \quad \mu=+,-
\end{aligned}
$$

consisting of the components

$I_{\mu \nu}{ }^{i s}$ propagating internal waves;

$F_{\mu \nu}{ }^{l j}$ contamination by current fine structure $(\mu, \nu=+,-)$;

$N_{\mu \nu}{ }^{l J}$ current noise;

$F_{00}{ }^{1 /}$ contamination by temperature fine structure.

These components are parameterized in the following way:

Internal waves. The cross-spectral matrix $I_{\mu \nu}{ }^{i j}(\omega)$ of a random internal wave field can be expressed in terms of the energy spectrum $E^{\sigma}(\alpha, \varphi, \omega)$ (cf. appendix). The spectrum is factorized in the form

$$
E^{\sigma}(\alpha, \varphi, \omega)=E^{\sigma}(\omega) A(\alpha ; \omega) S(\varphi ; \omega)
$$

where $E^{\sigma}(\omega)$ denotes the energy of upward ( $\sigma$ negative) and downward ( $\sigma$ positive) propagating waves of frequency $\omega ; A(\alpha$; $\omega)$ is the normalized wave number distribution, and $S(\varphi ; \omega)$ the normalized directional distribution. Following closely Garrett and Munk, we choose for the wave number distribution

$$
\begin{gathered}
A(\alpha ; \omega)=I(t, s)\left\{1+\left(\frac{\alpha-\alpha_{P}}{\alpha_{*}}\right)\right\}^{s t / s} \quad \alpha \geq \alpha_{P} \\
A(\alpha ; \omega)=0 \quad \alpha<\alpha_{P}
\end{gathered}
$$

where the normalization factor is given by

$$
I(t, s)=s \Gamma\left(\frac{t}{s}\right)\left\{\Gamma\left(\frac{1}{s}\right) \Gamma\left(\frac{t-1}{s}\right)\right\}^{-1}
$$

The distribution contains four parameters: a horizontal wave number scale $\alpha_{*}$, a low wave number cutoff $\alpha_{P}$, a high wave number slope $t$, and a parameter $s$ which describes the shape of the distribution at low wave numbers. The distribution always develops a peak at $\alpha_{P}$ which is pronounced if $s \rightarrow 1$ and flattens if $s \rightarrow \infty$. We shall refer to $\alpha_{p}$ and $s$ as the peak wave number and the peak shape parameter. The equivalent bandwidth $\alpha_{e}$ defined by (23) depends on $\alpha_{*}, t$, and $s$ :

$$
\alpha_{e}=\alpha_{*} \frac{\Gamma\left(\frac{1}{s}\right) \Gamma\left(\frac{2 t}{s}\right) \Gamma^{2}\left(\frac{t-1}{s}\right)}{s \Gamma\left(\frac{2 i-1}{s}\right) \Gamma^{2}\left(\frac{t}{s}\right)}
$$

Instead of $\alpha_{e}$ and $\alpha_{P}$ we may alternatively use the equivalent mode number $j_{e}$ and the peak mode number $j_{P}$, respectively.

The form (64) was chosen because of analytical and computational convenience. It models a distribution with a power law at high wave numbers and a more or less pronounced peak at low wave numbers. The wave number distributions of Garrett and Munk [1972, 1975] and Cairns and Williams [1976] are included for special values of $t, s, j_{p}$, and $j_{e}$ (cf. Table 1).

The directional distribution is parameterized by

$$
S(\varphi ; \omega)=\frac{\Gamma^{2}(p+1) 2^{2 p}}{2 \pi \Gamma(2 p+1)} \cos ^{2 p}\left(\frac{\varphi-\varphi_{0}}{2}\right)
$$

which models a beam with propagation direction $\varphi_{0}$ and a width determined by the beam width parameter $p$, or alternatively,

$$
q=\frac{p}{p+1}
$$

An isotropic distribution is then obtained for $q \rightarrow 0(p \rightarrow 0)$, a unidirectional distribution for $q \rightarrow 1(p \rightarrow \infty)$. The beamwidth $\Delta \varphi$ defined by

$$
\cos ^{2 p}\left(\frac{\Delta \varphi}{2}\right)=\frac{1}{2}
$$

depends almost linearly on $q$ (cf. paper 1).

Contamination by temperature fine structure. The contamination $F_{00}{ }^{1 j}$ of the displacement measurements by temperature fine structure is parameterized following McKean [1974]. McKean considered stationary and horizontally homogeneous temperature fine structure with a mean square temperature jump $\overline{\theta^{2}}$ over a mean layer thickness $d$, superimposed on a mean temperature gradient $d \bar{T} / d z$. If this temperature fine structure is vertically advected by internal waves with a (true) displacement spectrum

$$
P_{\zeta \zeta}(\omega)=\left\langle\zeta^{2}\right\rangle f \omega^{-2}
$$

temperature sensors at a fixed depth observe an additional displacement spectrum

$$
F_{\zeta \zeta}(\omega)=\delta_{f}(\omega) P_{\zeta \zeta}(\omega)
$$

where

$$
\delta_{f}(\omega)=\frac{1}{2} \frac{\overline{\theta^{2}}}{d \bar{T} / d z} \frac{(\omega / f)^{1 / 2}}{d\left(\zeta^{2}\right)^{1 / 2}}
$$

is the temperature fine structure ratio. The contamination of the displacement cross spectra is given by

$$
F_{\zeta \zeta}{ }^{\prime \prime}=\delta_{f}\left(P_{\zeta \zeta}{ }^{\prime \prime} P_{\zeta \zeta}{ }^{\prime \prime}\right)^{1 / 2} \Lambda(\Delta z)
$$

McKean's theory predicts the form

$$
\Lambda(\Delta z)=e^{-\Delta z / a}\left(\cos \frac{\Delta z}{a}+\sin \frac{\Delta z}{a}\right)
$$

for the fine structure coherence (normalized cospectrum) with a vertical coherence scale

$$
a(\omega)=\left\langle\zeta^{2}\right\rangle^{1 / 2}(\omega / f)^{-1 / 2}
$$

We parameterize the contamination of the displacement field by the functional form (73) with (74), taking as parameters the fine structure ratio $\delta_{f}$ and the coherence scale $a$. In McKean's theory both turn out to be frequency dependent.

The current contaminations. As was discussed above, the origin of the current contaminations cannot be inferred from the Iwex measurements, mainly because direct measurements of current fine structure are lacking. Here we suggest that the 

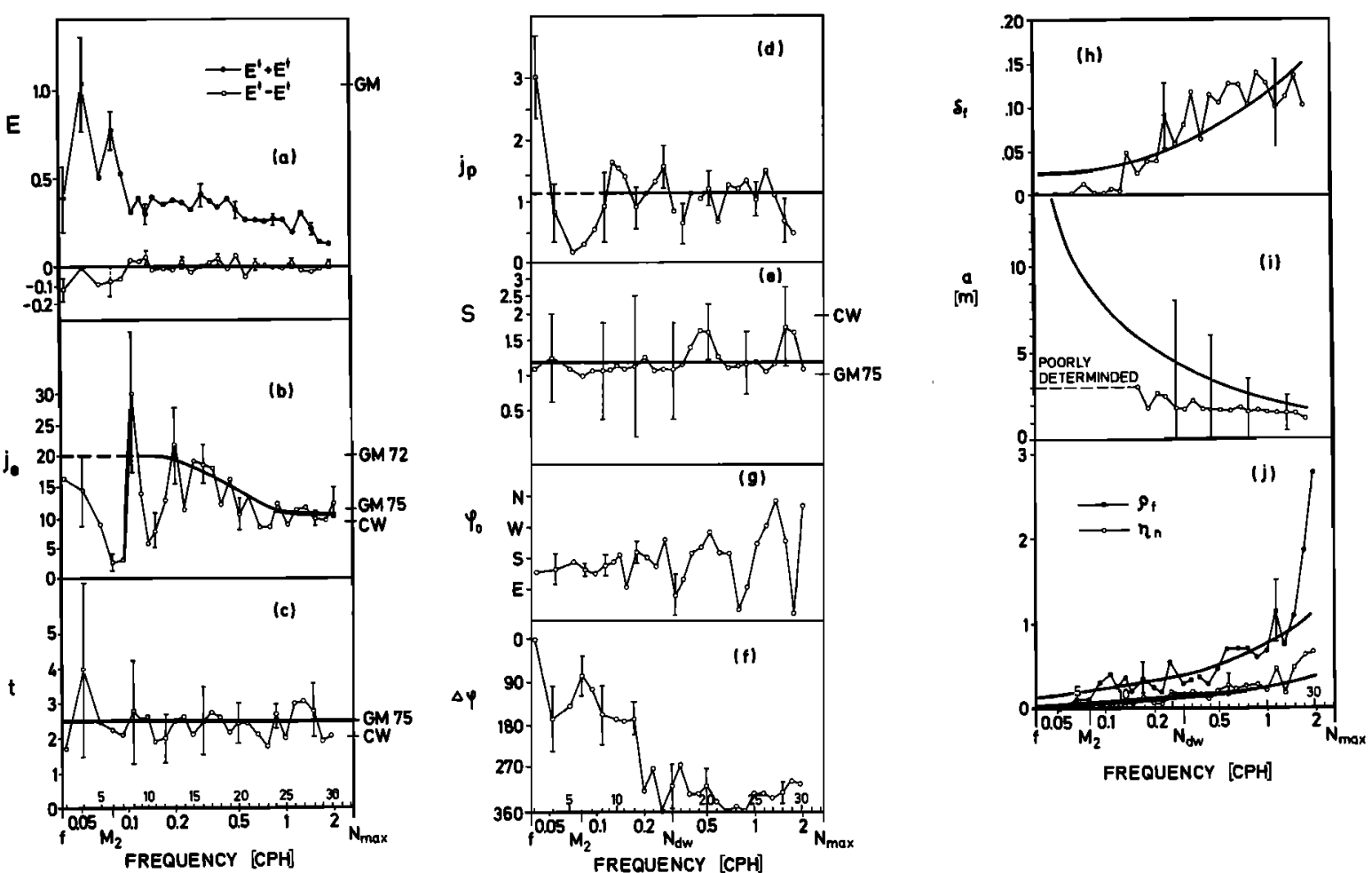

Fig. 15. Parameters of Iwex model versus frequency. Full curves indicate parameterizations of frequency dependence. The values proposed by Garrett and Munk [1972, 1975] and Cairns and Williams [1976] are indicated at the right border (they apply to the entire frequency range). The total wave energy and energy asymmetry (Figure 15a) are scaied by GM's frequency distribution $E_{0} \cdot B(\omega)$ (see $\left.(18), E_{0}=4 \cdot 10^{8} \mathrm{ergs} / \mathrm{cm}^{2}\right)$.

current field is contaminated by passive current fine structure advected by internal waves past the current meters and by current noise being of either geophysical or instrumental nature. Similar to the temperature fine structure contamination we parameterize the contribution of the current fine structure to the observed cross spectra by

$$
\begin{aligned}
& F_{\mu \nu}{ }^{l j}=\frac{1}{2} \delta_{\mu \nu} \rho_{f}\left\{\left(P_{++}{ }^{u}+P_{--}{ }^{u}\right)\left(P_{++}{ }^{j \prime}+P_{--}{ }^{j}\right)\right\}^{1 / 2} \Lambda(\Delta z) \\
& \mu, \nu=+,-
\end{aligned}
$$

where $\Lambda(\Delta z)$ is given by (74) with $a$ replaced by a corresponding current fine structure coherence scale. The Iwex data allow only the determination of the current fine structure ratio $\rho_{r}$. The spatial resolution of the array was insufficient to determine the fine structure coherence scale except that it must be smaller than the smallest vertical separation between current meters $(\approx 7 \mathrm{~m})$. This conclusion does not depend on the specific form of $\Lambda(\Delta z)$.

The contamination of the current measurements by current noise is parameterized by

$$
\begin{aligned}
N_{\mu \nu}{ }^{j}=\frac{1}{2} \delta_{\mu \nu} \eta_{n}\left\{\left(P_{++}{ }^{i}+P_{--}{ }^{l i}\right)\left(P_{++}{ }^{j}+P_{--}{ }^{j}\right)\right\}^{1 / 2} \\
\cdot \exp \left(-\frac{r^{2}+(\Delta z)^{2}}{2 b^{2}}\right) \quad \mu, \nu=+,-
\end{aligned}
$$

Again the Iwex data allow only to determine the noise energy ratio $\eta_{n}$. The coherence scale $b$ could not be obtained for the same reason as above.

The Iwex model is hence characterized by 12 free parameters at each frequency:

total energy $E^{+}+E^{-}$;

energy asymmetry $E^{+}-E^{-}$; equivalent bandwidth $\alpha_{e}$ (or $j_{e}$ );

peak wave number $\alpha_{P}$ (or $\left.j_{P}\right)$;

high wave number slope $t$

peak shape parameter $s$;

propagation direction $\varphi_{0}$;

beam width parameter $q$ (or $\Delta \varphi$ );

temperature fine structure ratio $\delta_{f}$;

vertical coherence scale of temperature fine structure $a$; current fine structure ratio $\rho_{f}$;

current noise ratio $\eta_{n}$.

More general models with a larger number of parameters have been tried, but additional parameters could not be determined accurately, according to criteria discussed in paper 1 . In introducing, for example, different bandwidths, peak wave numbers, etc., for the up and downward spectra it turned out that the asymmetric components of these parameters are not determined, mainly because the energy asymmetry is too small. Similarly, a wave number dependence of the directional distribution could not be determined. The values for the directional parameters are representative for low wave numbers where the main part of the energy is located.

For each frequency, values of the parameters are found by the least squares fit described in the previous section. The results are displayed in Figure 15 and discussed in detail below.

Energy (Figure 15a). The figure displays the total energy and the energy asymmetry normalized by the frequency distribution $E_{0} \cdot B(\omega)$ of GM's model (cf. equation (18), $E_{0}=4 \cdot 10^{\circ}$ ergs $/ \mathrm{cm}^{2}$ ). In the continuum the wave field is symmetric, and the frequency distribution of the total energy is well represented by a -2 power law. At inertial and tidal frequency the field is asymmetric; about $20 \%$ more energy propagates down- 
ward than upward. This agrees roughly with the findings of Leaman and Sanford [1975], who analyzed inertial motions with a dropped current meter. For our data a value of $E_{0}=10^{\circ}$ ergs $/ \mathrm{cm}^{2}$ is appropriate to model correctly the behavior in the continuum. Then the low-frequency energy exceeds the one of GM's $B(\omega)$ considerably. The unscaled spectrum is given in Figure 18 below.

Equivalent bandwidth (Figure $(5 b$ ). Displayed is the equivalent mode number $j_{e}$ calculated from the dispersion relation corresponding to the Iwex profile. As expected, the bandwidth varies considerably with frequency. We find extremely low values at the tidal frequency $M_{2}$ and also at the tenth, eleventh, fourteenth, and eighteenth frequencies. These lie close to the harmonics of $\boldsymbol{M}_{\mathbf{2}}$. In the internal wave continuum there seems to be a decrease of $j_{e}$ starting from about 20 modes just beyond $M_{2}$ down to about 10 modes at high frequencies. This behavior is well approximated by

$j_{e}=j_{\infty}+\frac{1}{2}\left(j_{0}-j_{\infty}\right)\left[1-\tanh \frac{\log \omega / f-\log \omega_{m} / f}{\frac{1}{4} \log \omega_{0} / \omega_{\infty}}\right]$

where $j_{\infty}=10$ is the mode number at high frequencies (attained approximately at $\omega_{\infty}=1.133 \mathrm{cph}$ ) and $j_{0}=20$ the mode number at low frequencies (from $\omega=f$ to approximately $\omega_{0}=$ $0.44 \mathrm{cph}$ ). At $\omega_{m}=0.173 \mathrm{cph}$ the mode number is $j_{e} \approx \frac{1}{2}\left(j_{0}+\right.$ $\left.j_{\infty}\right) \approx 15$. A decrease of the number of excited modes with frequency has also been found by Pinkel [1975].

The high wave number slope (Figure $15 \mathrm{c}$ ). This scatters less than $j_{e}$. At each single frequency the large error bars do not allow discrimination between Garrett and Munk's $t=2.5$ and Cairns and Williams' $t=2$. The average value

$$
t=2.4 \pm 0.4
$$

favors a model with $t$ larger than 2 .

The peak mode number (Figure 15d). Again, considerable variation with frequency is shown. At the inertial frequency we find no energy in the barotropic and first two baroclinic modes. The barotropic mode is excited at the tide but not in the continuum. Contrary to $j_{e}$ the downward pointing spikes in $j_{P}$ do not correspond to harmonics of the $M_{2}$ tide. The average value of $j_{P}$ in the continuum is

$$
j_{P}=1.2 \pm 0.3
$$

The peak shape parameter (Figure 15e). Here smooth behavior is shown. The mean value in the continuum

$$
s=1.2 \pm 0.4
$$

lies closer to $s=1$ of GM75 than to $s=2$ of Cairns and Williams, indicating that the Iwex data require a sharp peak at low wave numbers.

Beamwidth (Figure 15f). Only at low and to a lesser extent at high frequencies is the wave field significantly anisotropic. At low frequencies the beamwidth is about $90^{\circ}$.

The propagation direction (Figure $15 \mathrm{~g}$ ). This is a sensible parameter only if the field shows a strong directionality. At low frequencies, propagation toward the southeast is found, i.e., away from the continental shelf.

The fine structure ratio (Figure 15h). This value agrees remarkably well with the theoretical prediction of McKean [1974]. The smooth curve given by

$$
\delta_{f}(\omega)=0.021(\omega / f)^{1 / 2}
$$

is the prediction at 724-m depth, according to Joyce and Desaubies [1977]. They found $\bar{\theta}^{2}=95.3\left(\mathrm{~m}^{\circ} \mathrm{C}\right)^{2}, d \bar{T} / d z=17.7 \mathrm{~m}$ ${ }^{\circ} \mathrm{C} \mathrm{m}{ }^{-1}$, and $\left\langle\zeta^{2}\right\rangle^{1 / 2}=6.9 \mathrm{~m}$.
The fine structure coherence scale (Figure 15i). This parameter could not be determined at low frequencies, mainly because here $\delta_{f}$ is too small. In the continuum the values are in good agreement with McKean's theory. The smooth curve

$$
a(\omega)=6.9 \mathrm{~m} \cdot(\omega / f)^{-1 / 2}
$$

follows from (75).

Current fine structure and noise ratios (Figure 15j). Both ratios increase with frequency, the fine structure ratio being about 2-3 times larger than the noise ratio. At high frequencies the energy in the current contamination becomes comparable with the internal wave energy. The sharp increase at very high frequencies is presumably due to the inadequacy of the propagating wave model near the turning point. The smooth curves given by

$$
\begin{aligned}
& \rho_{f}(\omega)=0.15(\omega / f)^{1 / 2} \\
& \eta_{n}(\omega)=0.05(\omega / f)^{1 / 2}
\end{aligned}
$$

describe the mean behavior.

In a statistical sense the above set of parameters is not optimal because they are not completely uncorrelated. Correlations between various parameters are displayed in paper 1 . They are sufficiently small to guarantee that the naive interpretation suggested by their names is valid. Another choice of parameters may prevent this. For example, using the mode number scale $j_{*}$ instead of $j_{e}$ as a free parameter, we found a high correlation between $j_{*}$ and $t$. Hence these latter parameters describe similar features. Indeed, increasing $j_{*}$ with fixed $t$ or decreasing $t$ with fixed $j_{*}$ essentially reduces the coherence scale only (cf. Figure $8 b$ ). Therefore if we use $j_{*}$ as a parameter, $j_{*}$ may not be interpreted as bandwidth, nor may $t$ be interpreted as a mere shape parameter.

\section{Consistency of the Model}

It remains to demonstrate that the Iwex model with the parameter values specified above provides a consistent description of the data. A direct comparison of some observed and predicted coherences is shown in Figure 16. The predicted values lie within the standard deviation of the observations. An attempt to fit the data by a pure internal wave field is also shown in the figure. The best internal wave model is indeed unable to reproduce the observed coherences and behaves rather diplomatically, fitting none of them.

A unique decision to the consistency of the Iwex model is obtained by considering the distance $\epsilon$ between model and data point as defined in (34). The criterion (35) of accepting a model must be modified, since we used the modified weights

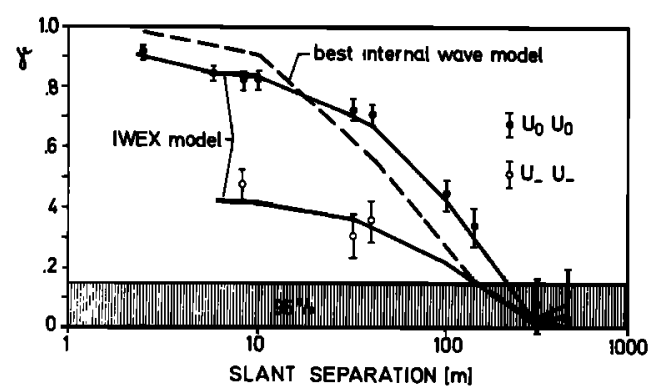

Fig. 16. Comparison of observed slant displacement and current coherences with model coherences at $\omega_{2 \theta} \triangleq 0.88$ hour. Dashed line, best internal wave model (applies to both coherences); solid lines, Iwex model. 
(61), and we have to consider that the parameters are random variables. The squared distance $\epsilon^{2}(x)$ now follows a nonnormalized $\chi^{2}$ distribution with mean

$$
\left\langle\epsilon^{2}\right\rangle=\operatorname{Tr} \mathbf{S W}-\operatorname{Tr} \mathbf{M R}
$$

and approximately $L_{\mathrm{eff}}-P$ degrees of freedom. We must hence reject the model if

$$
\frac{\epsilon^{2}}{\left\langle\epsilon^{2}\right\rangle}>\frac{\chi_{L_{\mathrm{efr}}-P, 0.00^{2}}}{L_{\mathrm{efr}}-P}
$$

Figure 17 shows $\epsilon^{2} /\left\langle\epsilon^{2}\right\rangle$ with its $95 \%$ confidence limits. The figure can be directly compared with the consistency test of the Iwex model class shown in Figure 13i. The two figures agree satisfactorily; deviations must be attributed to the fact that the inverse technique uses all data while the consistency tests are applied to a subset.

Except for the tide and very high frequencies the Iwex model agrees perfectly with the complete set of data. Observed and predicted values of all cross spectra are statistically indistinguishable. As has already been explained, the discrepancy at high frequencies can easily be removed by using Airy functions or standing modes instead of WKB solutions. At the tide an adequate model must have a deterministic, counterclockwise rotating, barotropic current.

\section{SUMmary AND CONCLUSIONS}

The following main results were obtained from our analysis of the Iwex data:

1. The observed fluctuations are mainly due to random linear internal waves.

2. The displacement measurements are contaminated by the advection of layered temperature fine structure past the sensors.

3. The current measurements are contaminated by current fine structure and current noise.

4. The internal wave field is horizontally isotropic and vertically symmetric except at inertial and tidal frequency.

5. The wave number spectrum is in good agreement with model GM75 except for a strong peak at low but nonzero waye number and a slight decrease of the mode number bandwidth with frequency.

6. At tidal frequency a description of the motion by random waves is not appropriate.

Figure $18 a$ shows the partition of the observed spectrum of horizontal kinetic energy (i.e., the sum of $\boldsymbol{P}_{++}$and $\boldsymbol{P}_{--}$from Figure 2) into its three components, namely, internal waves, current fine structure, and current noise. In contrast to temperature fine structure, where the interpretation is confirmed by independent measurements, the interpretation of the contamination as current 'fine structure' and current 'noise' is somewhat tentative. Additional information from separations smaller than $7 \mathrm{~m}$ and from dropped current meters is necessary to determine the origin of the current contamination.

Figure $18 b$ displays the partition of the observed displacement spectrum (cf. Figure $2 b$ ) into internal waves and the contamination contribution. It is again emphasized that the contamination signal does not correspond to vertical motions.

The analytical form (64) of the wave number distribution is somewhat arbitrary, and we do not claim it to be superior to other choices. However, most parameters are defined such that they represent basic properties of the spectrum, and their values are almost independent of the particular form (64). Any other model would have values for energy, bandwidth, high wave number slope, and peak wave number which are within the error bars of the values given in Figure 15.

The parameters of the model (cf. Figure 15) show considerable scatter with frequency. Some parameters scatter in a statistically insignificant way about a mean trend in the entire frequency range. Others show completely different behavior at low frequencies from that in the continuum; harmonics of the tide can be located particularly in the mode number bandwidth. Therefore parameterization of the frequency dependence seems to be sensible only in limited regimes.

The model describes the time-averaged state of the field. Conclusions about any instantaneous state, even of a qualitative kind, cannot be made, since features such as isotropy and symmetry may result from the averaging [Briscoe, 1975]. Other features, such as the contamination pattern and the wave number structure, are likely to apply also to any state.

The Iwex model is based on a large number of data ( $\approx 40,000$ cross-spectral values) which have been analyzed by two formal techniques. Consistency tests were applied to test such basic conceptions as the adequacy of the internal wave description, symmetry, and isotropy. The tests are easily performed by evaluating the statistic $\Delta^{2}$ (cf. equation (41)) and applying the likelihood criterion. While this method is well suited to finding inconsistencies between data and a particular model, it does not provide a systematic way to construct a model. The inverse technique is an indirect method to determine the wave number spectrum. Direct methods [e.g., Capon, 1969] are not appropriate for broadband spectra with the spatial resolution of this experiment. The inverse technique is conceptually straightforward, but its actual computations are lengthy and time-consuming for the large data set. The major computational problem is the numerical evaluation of the integral (cf. equation (A20)) of the internal wave model, which has to be done for each model cross spectrum separately. Special integration techniques have been employed to overcome computer time and accuracy problems. Compared to the simple reasoning in the first part of this paper, the consistency tests and the inverse analysis provide precise and quantitative results. We recommend applying the computationally simple and inexpensive consistency tests to any similar data set. They always give basic though not detailed information. The elaborate and costly inverse analysis should only be performed if the model class is proven to be consistent.

Since the inverse technique maps the information contained in the observed cross spectra onto a set of parameters, the question arises as to what observations contribute to what parameters. Because of the perfect triangular structure of the array every horizontal separation between sensors occurs three times (with different orientation). Comparison of the corre-

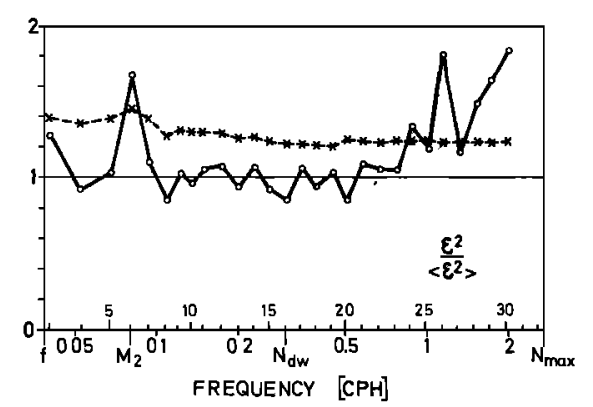

Fig. 17. Consistency of the Iwex model: normalized distance between data and model point with $95 \%$ confidence limit versus frequency. 

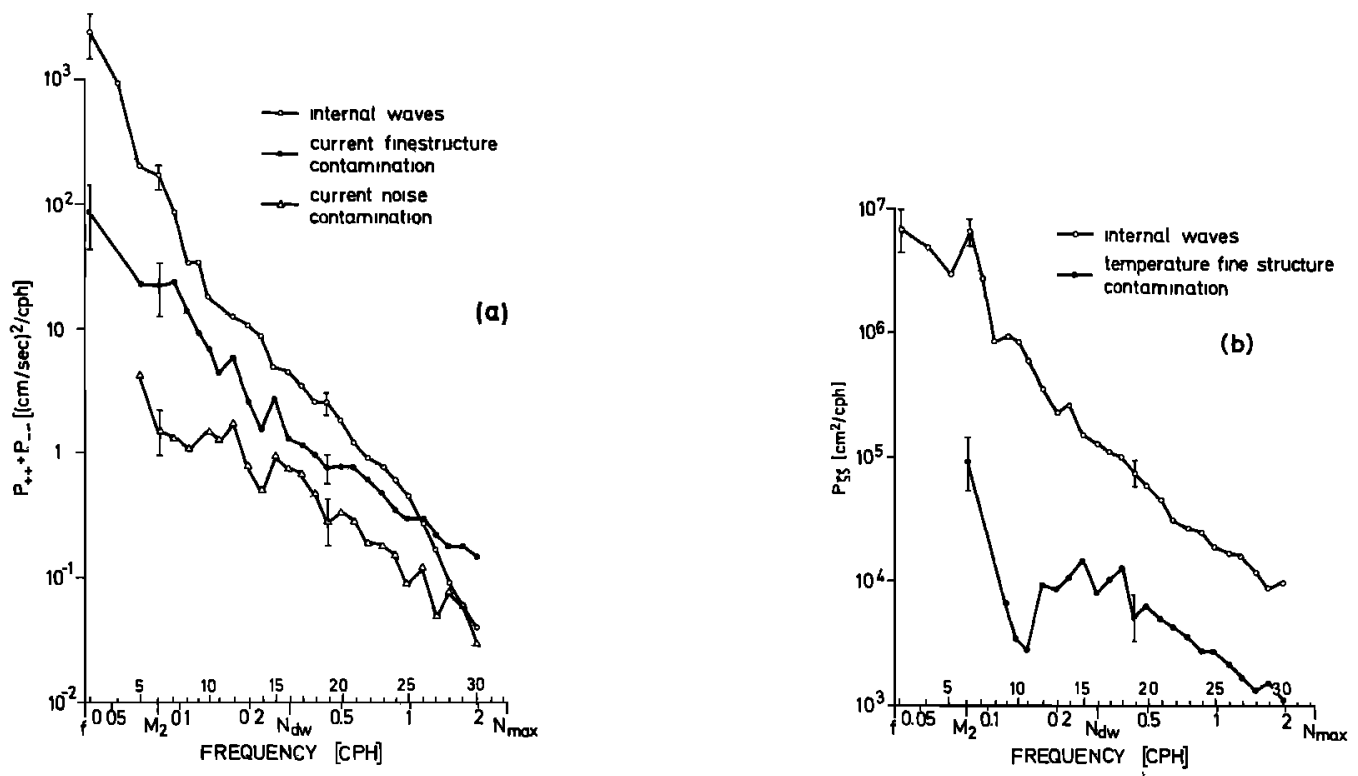

Fig. 18. Partition of the observed spectra into internal waves and contamination. (a) The spectra of horizontal kinetic energy of the internal wave part, the current fine structure, and the current noise part. (b) The displacement spectra of the internal wave part and the fine structure part.

sponding cross spectra only provides information about the directional properties of the field. For internal waves the horizontal current field is related to the vertical displacement. Comparison of both measurements allows the detection of contaminations. Similarly, horizontal separations are related to vertical (slant) ones by the dispersion relation. Comparison again only determines contaminations. The conceptually simplest array for determining the wave number spectrum of an isotropic and symmetric wave field would hence be a horizontal chain of temperature sensors. As is shown in the appendix, this expresses the formal fact that for an isotropic and symmetric internal wave field only 1 out of 18 components of the cross-spectral matrix between separated instruments is independent, for example, the displacement cospectrum. Adding current meters to the horizontal chain does not yield any additional information on the wave number spectrum but merely allows the determination of contamination by current noise (and gives some limited information about isotropy and symmetry). Adding a vertical chain of temperature sensors or current meters allows the detection of temperature or current fine structure. Thus a highly complex mooring such as Iwex is required for the determination of all details of our spectral model. If certain features are known a priori, less sophisticated moorings are sufficient. It is hoped that Iwex will enlarge the a priori knowledge for future experiments.

\section{NOTATION}

$u_{1}, u_{2}, u_{3}=u_{0} \quad$ Cartesian components of velocity vector.

$u_{+}, u_{-}$rotary components of horizontal velocity, defined in (3).

$\zeta$ vertical displacement.

$\omega$ frequency.

$f$ inertial frequency.

$M_{2}$ frequency of semidiurnal tide.

$N=N(z)$ local buoyancy frequency.

$\mathbf{r}_{i j}$ horizontal separation vector between $i$ th instrument and $j$ th instrument.

$\Delta z \quad$ vertical separation.

$r$ horizontal separation. $\alpha=(\alpha, \varphi)$ horizontal wave vector (polar coordinates, counterclock wise from east).

$\beta$ vertical wave number, $\beta=\alpha\left[\left(N^{2}-\omega^{2}\right) /\left(\omega^{2}-\right.\right.$ $\left.\left.f^{2}\right)\right]^{1 / 2}$.

$\theta_{l j}$ vertical phase difference.

$A_{\mu \nu}{ }^{i j} \quad$ cross spectrum between $u_{\mu}$ at $i$ th position and $u_{v}$ at $j$ th position.

$\gamma_{\mu \nu}{ }^{l j}, \Phi_{\mu \nu}{ }^{i s}$ coherence and phase.

$P_{\mu \nu}{ }^{l j}, Q_{\mu \nu}{ }^{i j}$ cospectrum and quadspectrum.

$I_{\mu \nu}{ }^{i s}$ cross-spectral matrix for internal wave model, defined in (A19).

\section{Appendix: Algebraic Structure of a Random INTERNAL WAVE FIELD}

\section{The Rotary Representation}

The moored current meters and temperature sensors of the Iwex experiment measure time series of the three velocity components at fixed points in space. The vertical velocity is inferred from temperature and temperature gradient. These time series are denoted by $u_{m}\left(x_{t}, t\right)$, where $m=1,2,3$ denotes the three velocity components $\left(u_{1}\right.$ eastward, $u_{2}$ northward, $u_{\mathrm{a}}$ upward $)$ and $\mathbf{x}_{i}(i=1, \cdots, 20)$ the position of the instruments.

As was pointed out in the introduction, it is more appropriate to describe the horizontal current in terms of its rotary components

$$
\begin{aligned}
& u_{ \pm}(\mathbf{x}, t)=\frac{1}{(2)^{1 / 2}}\left\{u_{1}(\mathbf{x}, t) \pm i u_{2}(\mathbf{x}, t)\right\} \\
& u_{0}(\mathbf{x}, t)=u_{3}(\mathbf{x}, t)
\end{aligned}
$$

The latter definition is introduced for convenience only. We will use $u_{-}\left(u_{+}\right)$to describe the clock wise (anticlock wise) rotating component of the horizontal current. The covariance function is defined by

$$
\begin{array}{r}
R_{\mu \nu}{ }^{l}(\tau)=\left\langle u_{\mu}^{*}\left(\mathrm{x}_{i}, t\right) u_{\nu}\left(\mathrm{x}_{j}, t+\tau\right)\right\rangle \\
\mu, \nu=+,-, 0
\end{array}
$$


and the cross-spectral matrix by

$$
\begin{aligned}
A_{\mu \nu}{ }^{i j}(\omega)=P_{\mu \nu}{ }^{i j}(\omega)-i Q_{\mu \nu}{ }^{i j} & \\
& =\frac{1}{\pi} \int_{-\infty}^{+\infty} d \tau R_{\mu \nu}{ }^{i j}(\tau) e^{-i \omega \tau}
\end{aligned}
$$

The 'reality condition,' $u_{v}(\mathbf{x}, t)=u_{-\nu}{ }^{*}(\mathbf{x}, t)$, implies

$$
A_{\mu \nu}{ }^{j}(-\omega)=\left[A_{-\mu-\nu}{ }^{l j}(\omega)\right]^{*}
$$

The stationarity condition implies

$$
A_{\mu \nu}{ }^{j j}(\omega)=A_{-\nu-\mu}^{j i}(-\omega)
$$

and hence we restrict ourselves to positive frequencies and $i \geq j$.

For $i=j$ we have the additional relations

$$
A_{\mu \nu}{ }^{\prime \prime}(\omega)=\left[A_{\mu \nu}{ }^{\prime \prime}(\omega)\right]^{*}
$$

The coherence and phase between the rotary components are defined by

$$
\begin{aligned}
& \gamma_{\mu \nu}{ }^{i j}=\left|A_{\mu \nu}{ }^{l}\right|\left(P_{\mu \mu}{ }^{l i} P_{\nu \nu}{ }^{j J}\right)^{-1 / 2} \\
& \Phi_{\mu \nu}{ }^{i j}=\operatorname{Arctan}\left(Q_{\mu \nu}{ }^{i j}, P_{\mu \nu}{ }^{i j}\right)
\end{aligned}
$$

General transformation formulae between cross spectra in rotary and Cartesian representation are given in paper 1 . In particular, for $i=j$ the coherences squared between the rotary components take the form

$$
\begin{gathered}
\gamma_{+-}{ }^{2}=\frac{A_{+-} A_{+-}{ }^{*}}{A_{++} A_{--}}=\frac{\left(P_{11}-P_{22}\right)^{2}+4 P_{12}{ }^{2}}{\left(P_{11}+P_{22}\right)^{2}+4 Q_{12}{ }^{2}} \\
\gamma_{+0}{ }^{2}=\frac{A_{+0} A_{+0}{ }^{*}}{A_{++} A_{00}}=\frac{\left(P_{13}-Q_{23}\right)^{2}+\left(P_{23}+Q_{13}\right)^{2}}{\left(P_{11}+P_{22}+2 Q_{12}\right) P_{33}} \\
\gamma_{-0}{ }^{2}=\frac{A_{-0} A_{-0}{ }^{*}}{A_{--} A_{00}}=\frac{\left(P_{13}+Q_{23}\right)^{2}+\left(P_{23}-Q_{13}\right)^{2}}{\left(P_{11}+P_{22}+2 Q_{12}\right) P_{33}}
\end{gathered}
$$

Fofonoff [1969] introduced the 'colinear coherence'

$$
C_{\imath}=\left[\frac{\left(P_{11}-P_{22}\right)^{2}+4 P_{12}^{2}}{\left(P_{11}+P_{22}\right)^{2}}\right]^{1 / 2}=\left[\frac{A_{+-} A_{+-}{ }^{*}}{\left(P_{11}+P_{22}\right)^{2}}\right]^{1 / 2}
$$

and the 'rotary coherence'

$$
C_{0}=\frac{2 Q_{12}}{P_{11}+P_{22}}=\frac{P_{++}-P_{--}}{P_{++}+P_{--}}
$$

Note that both quantities are neither coherences between the Cartesian components nor coherences between the rotary components. Gonella [1972] used the names 'ellipse stability' $E$ $=\gamma_{+-}{ }^{2}$ and 'rotary coefficient' $C_{R}=C_{0}$.

\section{The Representation of Propagating}

\section{Internal Waves}

In the WKB approximation the internal wave field can be represented by

$$
\begin{gathered}
u_{\mu}(\mathbf{x}, z, t)=\sum_{\sigma= \pm} \int_{f}^{N} d \omega \int d^{2} \alpha\left[a(\mathbf{q}) U_{\mu}(\mathbf{q}) \psi_{\mu}(\mathbf{q}, z)\right. \\
\cdot \exp [-i(\alpha \cdot \mathbf{x}-\omega t)]+a^{*}(\mathbf{q}) U_{-\mu}^{*}(\mathbf{q}) \psi_{-\mu}{ }^{*}(\mathbf{q}, z) \\
\quad \exp [i(\alpha \cdot \mathbf{x}-\omega t)] \quad \mu=+,-, 0
\end{gathered}
$$

For an explanation of the symbols see the notation section.

The vertical eigenfunctions $\left(\psi_{+}=\psi_{-}=(1 / \alpha)\left(d \psi_{0} / d z\right)\right)$ are explicitly given by

$$
\psi_{\mu}(\mathrm{q}, z)=\Psi_{\mu} e^{-i \sigma \theta(z)}
$$

with

$$
\begin{aligned}
\Psi_{+} & =C(\omega) \Omega^{-1 / 2}(z)[-i \sigma \Omega(z)] \\
\Psi_{-} & =C(\omega) \Omega^{-1 / 2}(z)[-i \sigma \Omega(z)] \\
\Psi_{0} & =C(\omega) \Omega^{-1 / 2}(z) \\
\Omega(z) & =\left(\frac{N^{2}(z)-\omega^{2}}{\omega^{2}-f^{2}}\right)^{1 / 2}=\frac{\beta(z)}{\alpha} \\
\theta(z) & =\int_{z^{0}}^{z} d z^{\prime} \beta\left(z^{\prime}\right)
\end{aligned}
$$

Here $\beta(z)$ is the local vertical wave number, $\theta(z)$ the vertical phase, and $z^{0}$ the turning depth, i.e., $N\left(z^{0}\right)=\omega$.

The normalization constant $C(\omega)$ is given by

$$
C(\omega)=\frac{1}{\omega}\left(\omega^{2}-f^{2}\right)^{1 / 4}\left[\int d z \frac{N^{2}(z)-f^{2}}{\left(N^{2}(z)-\omega^{2}\right)^{1 / 2}}\right]^{-1 / 2}
$$

where the integration is carried out between the turning points. The amplitude factors are given by

$$
\begin{aligned}
& U_{+}(\mathbf{q})=-\frac{i}{(2)^{1 / 2}}(\omega-f) e^{i \varphi} \\
& U_{-}(\mathbf{q})=-\frac{i}{(2)^{1 / 2}}(\omega+f) e^{-i \varphi} \\
& U_{0}(\mathbf{q})=\omega
\end{aligned}
$$

If the wave!field is statistically stationary and-horizontally homogeneous and if upward and downward propagating waves are uncorrelated, the wave amplitudes satisfy the orthogonality relations

$$
\begin{gathered}
\left\langle a(\mathbf{q}) a\left(\mathbf{q}^{\prime}\right)\right\rangle=0 \\
\left\langle a(\mathbf{q}) a^{*}\left(\mathbf{q}^{\prime}\right)\right\rangle=\frac{1}{2} \delta\left(\mathbf{q}-\mathbf{q}^{\prime}\right) E(\mathbf{q})
\end{gathered}
$$

The normalization constant (A15) is chosen so that $E(q)$ is the spectrum of the total energy per unit surface area

$$
E_{0}=\sum_{\sigma= \pm} \int_{f}^{N} d \omega \int d^{2} \alpha E(\mathbf{q})
$$

When the field representation (A12) and the orthogonality conditions (A17) are used, the cross-spectral matrix becomes

$$
\begin{array}{r}
I_{\mu \nu}{ }^{i j}(\omega)=\sum_{\sigma= \pm} \int d^{2} \alpha E(\mathbf{q}) U_{\mu}^{*}\left(\mathbf{q} U_{\nu}(\mathbf{q}) \Psi_{\mu}\left(\mathbf{q}, z^{i}\right) \Psi_{\nu}\left(\mathbf{q}, z^{j}\right)\right. \\
\cdot \exp \left[-i\left(\alpha r_{i j}+\sigma \theta_{i \jmath}\right)\right]
\end{array}
$$

where

$$
\theta_{\imath},=\theta\left(z^{\iota}\right)-\theta\left(z^{j}\right)
$$

is the vertical phase difference and

$$
\dot{r}_{i j}=\mathbf{x}^{\imath}-\mathbf{x}^{\jmath}
$$

is the horizontal separation of the instruments. The cross spectra represent weighted projections of the energy spectrum $\mathrm{E}(q)$ onto the frequency axis. Explicitly, (A19) takes the form

$$
\begin{aligned}
& I_{\mu \nu}{ }^{j}(\omega)=B_{\mu \nu} \sum_{\sigma= \pm} \int d^{2} \alpha E(\mathbf{q}) C_{\mu \nu} D_{\mu \nu}{ }^{l j} \\
& \cdot \exp \left[-i\left(\alpha \mathrm{r}_{i j}+\sigma \phi_{i j}\right)\right]
\end{aligned}
$$


with

$\left\{B_{\mu \nu}(\omega)\right\}$

$$
\begin{aligned}
& =\left[\begin{array}{lcc}
\frac{1}{2}(\omega-f)^{2} & \frac{1}{2}(\omega-f)(\omega+f) & \frac{i}{(2)^{1 / 2}} \omega(\omega-f) \\
\frac{1}{2}(\omega-f)(\omega+f) & \frac{1}{2}(\omega+f)^{2} & \frac{i}{(2)^{1 / 2}} \omega(\omega+f) \\
-\frac{i}{(2)^{1 / 2}} \omega(\omega-f) & -\frac{i}{(2)^{1 / 2}} \omega(\omega+f) & \omega^{2}
\end{array}\right] \\
& \left\{C_{\mu \nu}(\varphi)\right\}=\left[\begin{array}{lll}
1 & e^{-2 \ell \varphi} & e^{-l \varphi} \\
e^{2 t \varphi} & 1 & e^{l \varphi} \\
e^{l \varphi} & e^{-l \varphi} & 1
\end{array}\right] \\
& \left\{D_{\mu \nu}{ }^{1 j}(\omega)\right\}=C^{2}(\tilde{\omega})\left[\begin{array}{ccc}
\left(\Omega_{l} \Omega_{\jmath}\right)^{1 / 2} & \left(\Omega_{t} \Omega_{j}\right)^{1 / 2} & i \sigma\left(\frac{\Omega_{l}}{\Omega_{j}}\right)^{1 / 2} \\
\left(\Omega_{l} \Omega_{j}\right)^{1 / 2} & \left(\Omega_{l} \Omega_{\jmath}\right)^{1 / 2} & \left(\frac{\Omega_{i}}{\Omega_{j}}\right)^{1 / 2} \\
-i \sigma\left(\frac{\Omega_{j}}{\Omega_{l}}\right)^{1 / 2} & -i \sigma\left(\frac{\Omega_{j}}{\Omega_{l}}\right)^{1 / 2} & \left(\Omega_{l} \Omega_{\jmath}\right)^{-1 / 2}
\end{array}\right]
\end{aligned}
$$

where $\Omega_{t}=\Omega\left(z^{i}\right)$. The corresponding formulae for the Cartesian representation can be found in paper 1 .

\section{Scaling}

If we consider the $z$ dependence of the cross-spectral matrix for $i=j$, we find

$$
\left\{I_{\mu \nu}{ }^{i}\right\} \sim\left[\begin{array}{lll}
\Omega_{i} & \Omega_{i} & 1 \\
\Omega_{i} & \Omega_{i} & 1 \\
1 & 1 & \Omega_{i}{ }^{-1}
\end{array}\right]
$$

implying the scaling relations

$$
\begin{aligned}
I_{++}(z), I_{+-}(z), I_{--}(z) & \sim \Omega(z) \sim N(z) \\
I_{+0}(z), I_{-0}(z) & \sim \text { const } \\
I_{00}(z) \sim \Omega^{-1}(z) & \sim N^{-1}(z)
\end{aligned}
$$

The latter proportionalities hold if $\omega \ll N(z)$.

\section{Consistency Relations}

Not all of the cross-spectral components are independent. From (A20)-(A 23) the complete set (52a)-(52d) of linearly independent consistency relations can be inferred [Müller and Siedler, 1976]. These relations, which are satisfied for arbitrary energy spectra $E(q)$, define eight consistency relations (counting real and imaginary parts separately) and have been utilized to test whether or not the observed fluctuations represent a field of propagating waves. We have formulated the consistency relations as linear relations among the cross spectra, since this is convenient for algebraic manipulations. They take a simple form if expressed in terms of coherences and phases:

$$
\begin{aligned}
& \gamma_{--}{ }^{i j}=\gamma_{00}{ }^{i j} \quad \Phi_{--}{ }^{i j}=\Phi_{00}{ }^{i j} \\
& \gamma_{++}{ }^{i j}=\gamma_{00} \text { lj } \quad \Phi_{--}{ }^{\imath j}=\Phi_{00}{ }^{i j} \\
& \gamma_{+0} i j=\gamma_{0-}{ }^{i j} \quad \Phi_{+0}{ }^{i j}=\Phi_{0-}{ }^{i j} \\
& \gamma_{-0}{ }^{i j}=\gamma_{0+}{ }^{i j} \quad \Phi_{-0}^{i j}=\Phi_{0+}{ }^{i j}
\end{aligned}
$$

If $i=j$, the number of consistency relations reduces to four. In this case, $(52 a)$ predicts the ratio of the horizontal and the vertical kinetic energy [Fofonoff, 1969]:

$$
\frac{P_{++}+P_{--}}{P_{00}}=\frac{\omega^{2}+f^{2}}{\omega^{2}} \frac{N^{2}-\omega^{2}}{\omega^{2}-f^{2}}
$$

Relation (52b) predicts the ratio between the anticlockwise and clockwise rotating parts of the motion

$$
\frac{P_{++}}{P_{--}}=\left(\frac{\omega-f}{\omega+f}\right)^{2}
$$

Relation $(52 c)$ takes the form

$$
\gamma_{+0}=\gamma_{-0} \quad \Phi_{+0}=-\Phi_{-0}
$$

\section{Symmetry and Isotropy Relations}

Since the 18 cross spectra between each instrument pair are related by the 8 consistency relations $(52 a)-(52 d)$, only 10 of them are independent. It is convenient to express them in terms of 10 linearly independent moments which are defined (in complex notation) as

$$
\begin{aligned}
M_{m}{ }^{i j}(\omega)= & \frac{1}{2 \pi} \sum_{\sigma= \pm} \int d^{2} \alpha E(\mathbf{q}) \sigma^{-m} \exp [-i m \varphi] \\
& \cdot \exp \left[i\left(\alpha \mathbf{r}_{i j}+\sigma \theta_{i j}\right)\right] \quad m=0, \pm 1, \pm 2
\end{aligned}
$$

For $i=j$, only five of these moments are independent, since $M_{-m}{ }^{*}=M_{m}$. The explicit relations between $I_{\mu \nu}{ }^{i j}$ and $M_{m}{ }^{i j}$ are listed in paper 1 .

The significance of these moments can easily be interpreted for $i=j$. When polar coordinates are used, the normalized moments reduce to

$$
M_{m}(\omega)=\frac{1}{2 \pi} \sum_{\sigma= \pm} \int_{0}^{\infty} d \alpha \int_{0}^{2 \pi} d \varphi \sigma^{-m} E^{\sigma}(\alpha, \varphi, \omega)
$$

Decomposing the spectrum into its even and odd component,

$$
E^{e, \eta}(\alpha, \varphi, \omega)=E^{+}(\alpha, \varphi, \omega) \pm E(\alpha, \varphi, \omega)
$$

we obtain

$$
\begin{gathered}
M_{0}(\omega)=\int_{0}^{\infty} d \alpha c_{0}{ }^{e}(\alpha, \omega) \quad M_{1}(\omega)=\int_{0}^{\infty} d \alpha c_{1}{ }^{0}(\alpha, \omega) \\
M_{2}(\omega)=\int_{0}^{\infty} d \alpha c_{2}{ }^{e}(\alpha, \omega)
\end{gathered}
$$

where $c_{m}{ }^{p, 0}(\alpha, \omega)$ are the Fourier coefficients of the expansion

$$
E^{\rho, 0}(\alpha, \varphi, \omega)=\sum_{m=-\infty}^{+\infty} c_{m}^{\rho, 0}(\alpha, \omega) e^{i m \varphi}
$$

Hence cross spectra obtained from a single instrument only provide information about the Fourier coefficients $c_{0}{ }^{e}, c_{1}{ }^{0}$, and $c_{2}{ }^{\circ}$.

If the energy density spectrum $E(q)$ is vertically symmetric, i.e., independent of the sign of the vertical wave number, the odd component of the spectrum is zero, and we find $M_{1}=0$. If the energy density spectrum is horizontally isotropic, i.e., independent of the direction $\varphi$ of the horizontal wave number, the Fourier coefficients $C_{1}{ }^{0}$ and $C_{2}{ }^{e}$ are zero, and we find $M_{1}=0$ and $M_{2}=0$. In terms of the cross spectra the symmetry relations are given by

$$
I_{+0}=0 \quad I_{-0}=0
$$

and the isotropy relations by

$$
I_{+0}=0 \quad I_{-0}=0 \quad I_{+-}=0
$$


which in terms of coherences are equivalent to the conditions $\gamma_{+0}=\gamma_{-0}$ for symmetry and $\gamma_{+0}=\gamma_{-0}=\gamma_{+-}$for isotropy.

Similar to the case $i=j$, symmetry and isotropy relations may be constructed for separated instruments $(i \neq j)$. Because of their algebraic complexity the reader is referred to Müller and Siedler [1976] and paper 1 for the details. The main result is that for slanted separation only three out of eighteen cross spectra are independent; the only nonvanishing moments are

$$
\begin{array}{r}
\operatorname{Re}\left\{M_{0}^{i \jmath}\right\}=\int_{0}^{2 \pi} d \alpha E(\alpha, \omega) J_{0}\left(\alpha r_{i j}\right) \cos \left(\theta_{i j}\right) \\
\operatorname{Re}\left\{M_{-1}{ }^{i j} e^{-i \psi}\right\}=-\int_{0}^{\infty} d \alpha E(\alpha, \omega) J_{1}\left(\alpha r_{\imath \jmath}\right) \sin \left(\theta_{i \jmath}\right) \\
\operatorname{Re}\left\{M_{-2}^{i j} e^{-2 i \psi}\right\}=-\int_{0}^{\infty} d \alpha E(\alpha, \omega) J_{2}\left(\alpha r_{i j}\right) \cos \left(\theta_{i j}\right)
\end{array}
$$

Here $\psi$ denotes the horizontal direction of the separation vector $\mathbf{r}_{i j}$. The moment (A40) models the spatial behavior of the coherences $\gamma_{++}{ }^{i}, \gamma_{--}{ }^{i j}$, and $\gamma_{00}{ }^{i j}$. The moments (A41) and (A42) determine the behavior of $\gamma_{+0}{ }^{i j}, \gamma_{0+}{ }^{i j}, \gamma_{-0} i j, \gamma_{0-}{ }^{i j}$, and $\gamma_{+-}$" and the corresponding phases. In the particularly interesting case of broadband spectra these latter coherences are always close to zero (equal to zero for $i=j$ ). The corresponding observed coherences will not be distinguishable from zero.

\section{Standing Internal Wave Modes}

For standing modes the vertical eigenfunctions are

$$
\psi_{+}^{(n)}=\psi_{-}^{(n)}=\frac{1}{\alpha} \frac{d \psi_{0}^{(n)}}{d z}
$$

where $\psi_{0}{ }^{n}(z)$ is the $n$th eigenfunction of

$$
\frac{d^{2} \psi_{0}}{d z^{2}}+\alpha^{2} \frac{N^{2}(z)-\omega^{2}}{\omega^{2}-f^{2}} \psi_{0}=0
$$

with boundary conditions $d \psi_{0} / d z=\left[g \alpha^{2} /\left(\omega-f^{2}\right)\right] \psi_{0}$ at $z=0$ and $\psi_{0}=0$ at $z=-h$.

The expression (A20) for the cross-spectral matrix must then be replaced by

$$
\begin{aligned}
& I_{\mu \nu}{ }^{1 j}(\omega)=B_{\mu \nu} \sum_{n=0}^{\infty} \int_{0}^{2 \pi} d \varphi E^{(n)}(\omega, \varphi) C_{\mu \nu} \tilde{D}_{\mu \nu}{ }^{j j} \\
& \cdot \exp \left[-i \boldsymbol{\alpha}^{(n)} \mathbf{r}_{i j}\right]
\end{aligned}
$$

Here $B_{\mu \nu}$ and $C_{\mu \nu}$ are defined by (A21) and (A22), while

$$
\tilde{D}_{\mu \nu}{ }^{l j}=\psi_{\mu}^{(n)}\left(z^{l}\right) \psi_{\nu}^{(n)}\left(z^{j}\right)
$$

The consistency relations for standing modes are given by (52e) and (52f) for horizontal separations and by $(52 b)$ for arbitrary separations. The isotropy relations can be found in paper 1 .

Acknowledgments. Thanks are expressed to all who made Iwex possible. We are particularly grateful to $\mathrm{M}$. Briscoe, who made the cross-spectral data set available to us. We have benefited greatly from discussions with $M$. Briscoe, C. Frankignoul, K. Hasselmann, T. Joyce, M. Noble, and F. Schott. The cooperation of the Rechenzentrum der Universität Kiel is appreciated. The hospitality of the Geophysical Fluid Dynamics Laboratory at Princeton University during the writing of the final version of this paper is gratefully acknowledged. We would like to thank Susan Euteneuer for typing the various manuscripts. Two of the authors (P.M. and D.J.O.) are supported through the Sonderforschungsbereich 'Meeresforschung Hamburg' by the Deutsche Forschungsgemeinschaft.

\section{REFERENCES}

Abramowitz, M., and I. A. Stegun (Eds.), Handbook of Mathematical Functions, Dover, New York, 1972.

Amos, D. E., and L. H. Koopmans, Tables of the distribution of the coefficient of coherence for stationary bivariant Gaussian processes, Rep. SCR-483. Sandia Corp., Albuquerque, N. Mex., 1963.

Briscoe, M. G., Preliminary results from the trimoored internal wave experiment (Iwex), J. Geophys. Res., 80(27), 3872-3884, 1975.

Briscoe, M. G., Gaussianity of internal waves, J. Geophys. Res., 82, 2117-2126, 1977.

Cairns, J. L.. Internal wave measurements from a midwater float, $J$. Geophys. Res., 80(3), 299-306, 1975.

Cairns, J. L., and G. O. Williams, Internal wave observations from a midwater float, 2, J. Geophys. Res., 81(12), 1943-1950, 1976.

Capon, J., High-resolution frequency-wavenumber spectrum analysis, Proc. IEEE, 57(8), 1408-1418, 1969.

Desaubies, Y. J. F., Internal waves near the turning point, Geophys. Fluid Dyn., 5, 143-154, 1973.

Desaubies, Y. J. F., A linear theory of internal wave spectra and coherences near the Väisälä frequency, J. Geophys. Res., 80(6), 895-899, 1975.

Eriksen, C. C., Measurements and models of finestructure, internal gravity waves and wave-breaking in the deep ocean, Ph.D. thesis, Mass. Inst. of Technol., Cambridge, and Woods Hole Oceanogr. Inst., Woods Hole, Mass., 1976.

Fofonoff, N. P., Spectral characteristics of internal waves in the ocean, Deep Sea Res., 16, suppl., 58-71, 1969.

Garrett, C. J. R., and W. H. Munk, Internal wave spectra in the presence of fine-structure, J. Phys. Oceanogr., I, 196-202, 1971.

Garrett, C. J. R., and W. H. Munk, Space-time scales of internal waves, Geophys. Fluid. Dyn., 2, 225-264, 1972.

Garrett, C. J. R., and W. H. Munk, Space-time scales of internal waves: A progress report, J. Geophys. Res., 80(3), 291-297, 1975.

Gonella, J., A rotary-component method for analysing meteorological and oceanographic vector time series, Deep Sea Res., 19, 833-846, 1972.

Goodman, N. R., On the joint estimation of the spectra, cospectrum and quadrature spectrum of a two-dimensional stationary Gaussian process, Sci. Pap. 10. Eng. Statist. Lab., N. Y. Univ., New York, 1957.

Hayes, S. P., Preliminary measurements of the time-lagged coherence of vertical temperature profiles, J. Geophys. Res., 80(3), 307-311, 1975.

Hayes, S. P., T. M. Joyce, and R. C. Millard, Jr., Measurements of vertical fine structure in the Sargasso Sea, J. Geophys. Res., 80(3), 314-319, 1975.

Hendry, R. M., The generation, energetics and propagation of internal tides in the western North Atlantic Ocean, Ph.D. thesis, Mass. Inst. of Technol., Cambridge, and Woods Hole Oceanogr. Inst., Woods Hole, Mass., 1975.

Jackson, D. D., Interpretation of inaccurate, insufficient and inconsistent data, Geophys. J. Roy. Astron. Soc., 28, 97-109, 1972.

Jenkins, G. M., and D. G. Watts, Spectral Analysis and Its Applications, 541 pp., Holden Day, San Francisco, Calif., 1968.

Joyce, T. M., Fine-structure contamination of moored temperature sensors: A numerical experiment, J. Phys. Oceanogr., 4, 183-190, 1974.

Joyce, T. M., and Y. J. F. Desaubies, Discrimination between internal waves and temperature finestructure, J. Phys. Oceanogr., 7, 22-32, 1977.

Katz, E. J., Tow spectra from Mode, J. Geophys. Res., 80(9), 1163-1 167, 1975.

Leaman, K., and T. Sanford, Vertical energy propagation of inertial waves: A vector spectral analysis of velocity profiles, J. Geophys. Res., 80(15), 1975-1978, 1975.

McKean, R. S., Interpretation of internal wave measurements in the presence of fine structure, J. Phys. Oceanogr., 4, 200-213, 1974.

Millard, R., Further comments on vertical temperature spectra in the Mode region, Mode Hot Line News, 18, 1, 1972.

Müller, P., and G. Siedler, Consistency relations for internal waves, Deep Sea Res., 23, 613-628, 1976.

Noble, N. A., and T. M. Joyce, Internal tidal observations during Iwex, submitted to Deep Sea Res., 1977.

Nuttall, A. H., Spectral estimation by means of overlapped fast Fourier transform processing of windowed data, NUSC Rep. 4169, Nav. Underwater Syst. Center, New London, Conn., 1971. 
Olbers, D. J., P. Müller, and J. Willebrand, Inverse technique analysis of a large data set, Phys. Earth Planet. Interiors, 12, 248-252, 1976.

Perrson, J., Variability and covariability of modified spectral estimates, IEEE Trans. Acoust. Speech Signal Process., ASSP-22. $158-160,1974$.

Phillips, O. M., On spectra measured in an undulating layered medium, J. Phys. Oceanogr., I, 1-6, 1971.

Pinkel, R., Upper ocean internal wave observations from Flip, $J$. Geophys. Res., 80(27), 3892-3910, 1975.

Sanford, T. B., Observations of the vertical structure of internal waves, J. Geophys. Res., 80(27), 3861-3871, 1975.

Schott, F., and J. Willebrand, On the determination of internal wave directional spectra from moored instruments, J. Mar. Res., 31, 116-134, 1973.

Tarbell, S., M. G. Briscoe, and D. Chausse, A compilation of moored current data and associated oceanographic observations, vol. IX,
1973 Internal Wave Experiment (Iwex), Tech. Rep. 75-68, Woods Hole Oceanogr. Inst., Woods Hole, Mass., 1976.

Webster, T. F., Estimates of the coherence of ocean currents over vertical distances, Deep Sea Res., 19, 35-44, 1972.

Wiggins, R., The general linear inverse problem: Implications of surface waves and free oscillations for earth structure, Rev. Geophys. Space Phys., 10(1), 25I, 1972.

Willebrand, J., P. Müller, and D. J. Olbers, Inverse analysis of the trimoored Internal Wave Experiment (Iwex), Ber. Inst. Meereskunde, 20a, $b, 222 \mathrm{pp} ., 1977$.

Zetler, B., W. Munk, H. Mofjeld, W. Brown, and F. Dormer, Mode tides, J. Phys. Oceanogr., 5, 430-441, 1975.

(Received April 1, 1977; revised July 1, 1977; accepted July 8,1977 .) 